\title{
Genome-wide association and functional studies identify 46 novel loci for alcohol consumption and suggest common genetic mechanisms with neuropsychiatric disorders
}

Evangelos Evangelou ${ }^{1,2} *$, He Gao ${ }^{1,3 *}$, Congying $\mathrm{Chu}^{4} *$, Georgios Ntritsos ${ }^{2}$, Paul Blakeley ${ }^{1,5}$, Andrew R Butts ${ }^{6}$, Raha Pazoki ${ }^{1}$, Hideaki Suzuki ${ }^{7,8}$, Fotios Koskeridis ${ }^{2}$, Andrianos M Yiorkas ${ }^{9,10}$, Ibrahim Karaman ${ }^{1,11}$, Joshua Elliott ${ }^{1}$, Stefanie Aeschbacher ${ }^{12}$, Traci M Bartz ${ }^{13,14}$, Sebastian E Baumeister ${ }^{15,16}$, Peter S Braund ${ }^{17,18}$, Michael R Brown ${ }^{19}$, Jennifer A Brody ${ }^{13}$, Toni-Kim Clarke ${ }^{20}$, Niki Dimou ${ }^{2}$, Jessica D Faul $^{21}$, Georg Homuth ${ }^{22}$, Anne U Jackson ${ }^{23}$, Katherine A Kentistou ${ }^{24,25}$, Peter K Joshi $^{24}$, Rozenn N Lemaitre ${ }^{13}$, Penelope A Lind ${ }^{26}$, Leo-Pekka Lyytikäinen ${ }^{27-29}$, Massimo Mangino ${ }^{30,31}$, Yuri Milaneschi ${ }^{32}$, Christopher P Nelson ${ }^{17,18}$, Ilja M Nolte ${ }^{33}$, Mia-Maria Perälä ${ }^{34,35}$, Ozren Polasek ${ }^{36}$, David Porteous ${ }^{37,38}$, Scott M Ratliff ${ }^{39}$, Jennifer A Smith ${ }^{21,39}$, Alena Stančákováa ${ }^{40}$, Alexander Teumer ${ }^{15,41}$, Samuli Tuominen ${ }^{42}$, Sébastien Thériault ${ }^{43,44}$, Jagadish Vangipurapu ${ }^{40}$, John B Whitfield ${ }^{45}$, Alexis Wood ${ }^{46}$, Jie $\mathrm{Yao}^{47}$, Bing $\mathrm{Yu}^{19}$, Wei Zhao ${ }^{39}$, Dan E Arking ${ }^{48}$, Juha Auvinen ${ }^{49,50}$, Chunyu Liu ${ }^{51}$, Minna Männikkö ${ }^{52}$, Lorenz Risch ${ }^{53-55}$, Jerome I Rotter ${ }^{56}$, Harold Snieder ${ }^{33}$, Juha Veijola $^{57-59}$, Alexandra I Blakemore ${ }^{9,10}$, Michael Boehnke ${ }^{23}$, Harry Campbell ${ }^{24}$, David Conen $^{43}$, Johan G Eriksson ${ }^{60-62}$, Hans J Grabe ${ }^{63,64}$, Xiuqing Guo ${ }^{47}$, Pim van der Harst $^{65-67}$, Catharina A Hartman ${ }^{68}$, Caroline Hayward ${ }^{69}$, Andrew C Heath ${ }^{70}$, MarjoRiitta Jarvelinn $^{71-75}$, Mika Kähönen ${ }^{76,77}$, Sharon LR Kardia ${ }^{39}$, Michael Kühne ${ }^{12}$, Johanna Kuusisto $^{78}$, Markku Laakso ${ }^{78}$, Jari Lahti ${ }^{42}$, Terho Lehtimäki ${ }^{27,28}$, Andrew M McIntosh $^{20,38}$, Karen L Mohlke ${ }^{79}$, Alanna C Morrison ${ }^{19}$, Nicholas G Martin ${ }^{45}$, Albertine J Oldehinkel ${ }^{68}$, Brenda WJH Penninx ${ }^{32}$, Bruce M Psaty ${ }^{80,81}$, Olli T Raitakari $^{82,83}$, Igor Rudan ${ }^{24}$, Nilesh J Samani ${ }^{17,18}$, Laura J Scott ${ }^{23}$, Tim D Spector ${ }^{30}$, Niek Verweij ${ }^{65}$, David R Weir ${ }^{21}$, James F Wilson ${ }^{24,69}$, Daniel Levy ${ }^{84,85}$, Ioanna Tzoulaki $^{1-3}$, Jimmy D Bell ${ }^{86 *}$, Paul Matthews ${ }^{7,11} *$, Adrian Rothenfluh ${ }^{6,87} *$, Sylvane Desrivières $^{4 *}$, Gunter Schumann ${ }^{4} *$, Paul Elliott ${ }^{1,3,11,88,89} *$ †

* Equal contribution

$\ddagger$ Corresponding authors

1. Department of Epidemiology and Biostatistics, Imperial College London, London, UK.

2. Department of Hygiene and Epidemiology, University of Ioannina Medical School, Ioannina, Greece.

3. MRC-PHE Centre for Environment and Health, Imperial College London, London, UK.

4. Medical Research Council-Social, Genetic and Developmental Psychiatry Centre, Institute of Psychiatry, Psychology \& Neuroscience, King's College London, London SE5 8AF, United Kingdom.

5. NIHR Imperial Biomedical Research Centre, ITMAT Data Science Group, Imperial College London, UK.

6. Molecular Medicine; School of Medicine; University of Utah, Salt Lake City, UT. 
7. Centre for Restorative Neurosciences, Division of Brain Sciences, Department of Medicine, Hammersmith Campus, Imperial College London, Du Cane Raod, London, W12 0NN, UK.

8. Tohoku Medical Megabank Organization, Tohoku University, 2-1 Seiryo, Aoba, Sendai, 980-8573, Japan.

9. Department of Life Sciences, Brunel University London, London, UK.

10. Section of Investigative Medicine, Imperial College London, London, UK.

11. UK Dementia Research Institute, Imperial College London, London, United Kingdom.

12. Cardiology Division, University Hospital Basel, 4031 Basel, Switzerland.

13. Cardiovascular Health Research Unit, Department of Medicine, University of Washington, Seattle, Washington, USA.

14. Department of Biostatistics, University of Washington, Seattle, WA, USA.

15. Institute for Community Medicine, University Medicine Greifswald, 17475 Greifswald, Germany.

16. Chair of Epidemiology, Ludwig-Maximilians-Universitat Munchen, UNIKAT Augsburg, 86156 Augsburg, Germany.

17. Department of Cardiovascular Sciences, University of Leicester, Cardiovascular Research Centre, Glenfield Hospital, Leicester, LE3 9QP, UK.

18. NIHR Leicester Biomedical Research Centre, Glenfield Hospital, Groby Road, Leicester, LE3 9QP, UK.

19. Human Genetics Center, Department of Epidemiology, Human Genetics \& Environmental Sciences, School of Public Health, The University of Texas Health Science Center at Houston, Houston, TX 77030 USA.

20. Department of Psychiatry, University of Edinburgh, Edinburgh, UK, EH10 5HF.

21. Survey Research Center, Institute for Social Research, University of Michigan, Ann Arbor, MI, USA.

22. Interfaculty Institute for Genetics and Functional Genomics, University Medicine Greifswald, 17475 Greifswald, Germany.

23. Department of Biostatistics and Center for Statistical Genetics, University of Michigan, Ann Arbor, Michigan, USA.

24. Centre for Global Health Research, Usher Institute for Population Health Sciences and Informatics, University of Edinburgh, Edinburgh, EH8 9AG, UK.

25. Centre for Cardiovascular Sciences, Queens Medical Research Institute, University of Edinburgh, Edinburgh, EH16 4TJ, Scotland.

26. Psychiatric Genetics, QIMR Berghofer Medical Research Institute, Brisbane, QLD 4006, Australia.

27. Department of Clinical Chemistry, Fimlab Laboratories, Tampere 33520, Finland.

28. Department of Clinical Chemistry, Finnish Cardiovascular Research Center Tampere, Faculty of Medicine and Life Sciences, University of Tampere, Tampere 33014, Finland.

29. Department of Cardiology, Heart Center, Tampere University Hospital, Tampere 33521, Finland. 
30. Department of Twin Research and Genetic Epidemiology, Kings College London, London SE1 7EH, UK.

31. NIHR Biomedical Research Centre at Guys and St Thomas Foundation Trust, London SE1 9RT, UK.

32. Department of Psychiatry, Amsterdam Neuroscience and Amsterdam Public Health research institute, Amsterdam University Medical Center, 1081 HJ Amsterdam, the Netherlands.

33. Department of Epidemiology, University Medical Center Groningen, University of Groningen, The Netherlands.

34. Folkhälsan Research Center, 00290 Helsinki, Finland.

35. Department of Public Health Solutions, National Institute for Health and Welfare, 00271 Helsinki, Finland.

36. Faculty of Medicine, University of Split, Split, 21000, Croatia.

37. Generation Scotland, Medical Genetics Section, Centre for Genomic and Experimental Medicine, Institute of Genetics and Molecular Medicine, University of Edinburgh, Edinburgh, UK, EH4 2XU.

38. Centre for Cognitive Ageing and Cognitive Epidemiology, Edinburgh, UK, EH8 9JZ.

39. Department of Epidemiology, University of Michigan, Ann Arbor, MI, USA.

40. Institute of Clinical Medicine, Internal Medicine, University of Eastern Finland, 70210 Kuopio, Finland.

41. DZHK (German Centre for Cardiovascular Research), partner site Greifswald, 17475 Greifswald, Germany.

42. Department of Psychology and Logopedics, Faculty of Medicine, 00014 University of Helsinki, Helsinki, Finland.

43. Population Health Research Institute, McMaster University, L8L 2X2 Hamilton, Canada.

44. Department of Molecular Biology, Medical Biochemistry and Pathology, Laval University, G1V 0A6 Quebec City, Canada.

45. Genetic Epidemiology, QIMR Berghofer Medical Research Institute, Brisbane, QLD 4006, Australia.

46. Department of Pediatrics/Nutrition, Baylor College of Medicine, 1 Baylor Plaza, Houston, Texas 77030 USA.

47. The Institute for Translational Genomics and Population Sciences, Department of Pediatrics, Los Angeles Biomedical Research Institute at Harbor-UCLA Medical Center, Torrance, California 90502, USA.

48. McKusick-Nathans Institute of Genetic Medicine, Johns Hopkins University School of Medicine, Baltimore, Maryland, USA.

49. Center for Life Course Health Research, Faculty of Medicine, University of Oulu, 90220, Oulu, Finland.

50. Oulunkaari Health Center, $91100 \mathrm{Ii}$, Finland.

51. Department of Biostatistics, Boston University School of Public Health.

52. Northern Finland Birth Cohorts, Faculty of Medicine, University of Oulu, 90220, Oulu, Finland.

53. Institute of Clinical Chemistry, Inselspital Bern, University Hospital, University of Bern, 3010 Bern, Switzerland. 
54. Labormedizinisches Zentrum Dr. Risch, Vaduz, Liechtenstein.

55. Private University of the Principality of Liechtenstein, Triesen, Liechtenstein.

56. The Institute for Translational Genomics and Population Sciences, Departments of Pediatrics and Medicine, Los Angeles Biomedical Research Institute at Harbor-UCLA Medical Center, Torrance, California 90502, USA.

57. Department of Psychiatry, Research Unit of Clinical Neuroscience, 90014 University of Oulu, Finland.

58. Department of Psychiatry, University Hospital of Oulu, 90220 Oulu, Finland.

59. Medical research Center Oulu, University and University Hospital of Oulu, 90220 Oulu, Finland.

60. Department of General Practice and Primary health Care, Tukholmankatu 8 B , 00014 University of Helsinki, Finland.

61. National Institute for Health and Welfare, 00271 Helsinki, Finland.

62. Helsinki University Central Hospital, Unit of General Practice, 00290 Helsinki, Finland.

63. Department of Psychiatry and Psychotherapy, University Medicine Greifswald, 17475 Greifswald, Germany.

64. German Center for Neurodegenerative Diseases (DZNE), Rostock/Greifswald, 17475 Greifswald, Germany.

65. University Medical Center Groningen, University of Groningen, Department of Cardiology, the Netherlands.

66. University of Groningen, University Medical Center Groningen, Department of Genetics, Groningen, The Netherlands.

67. Durrer Center for Cardiogenetic Research, ICIN-Netherlands Heart Institute, Utrecht, The Netherlands.

68. Department of Psychiatry, University Medical Center Groningen, University of Groningen, 9713 GZ Groningen, The Netherlands.

69. MRC Human Genetics Unit, Institute of Genetics and Molecular Medicine, University of Edinburgh, Western General Hospital, Crewe Road, Edinburgh, EH4 2XU, UK.

70. Department of Psychiatry, School of Medicine, Washington University in St. Louis, St. Louis, MO 63110, USA.

71. Department of Epidemiology and Biostatistics, MRC-PHE Centre for Environment and Health, School of Public Health, Imperial College London, London, W2 1PG, United Kingdom.

72. Center for Life Course Health Research, Faculty of Medicine, University of Oulu, PO Box 8000, FI-90014 Oulun yliopisto, Finland.

73. Biocenter Oulu, University of Oulu, Aapistie 5, 90220 Oulu, Finland.

74. Unit of Primary Health Care, Oulu University Hospital, OYS, Kajaanintie 50, 90220 Oulu, Finland.

75. Department of Life Sciences, College of Health and Life Sciences, Brunel University London, Kingston Lane, Uxbridge, Middlesex UB8 3PH, United Kingdom.

76. Department of Clinical Physiology, Tampere University Hospital, Tampere 33521, Finland. 
77. Department of Clinical Physiology, Finnish Cardiovascular Research Center Tampere, Faculty of Medicine and Life Sciences, University of Tampere, Tampere 33014, Finland.

78. Institute of Clinical Medicine, Internal Medicine, University of Eastern Finland, and Kuopio University Hospital, 70210 Kuopio, Finland.

79. Department of Genetics, University of North Carolina, Chapel Hill, NC, USA.

80. Cardiovascular Health Research Unit, Departments of Medicine, Epidemiology, and Health Services, University of Washington, Seattle, WA, 98101.

81. Kaiser Permanente Washington Health Research Institute, Seattle, WA, 98101.

82. Department of Clinical Physiology and Nuclear Medicine, Turku University Hospital, Turku 20521, Finland.

83. Research Centre of Applied and Preventive Cardiovascular Medicine, University of Turku, Turku 20014, Finland.

84. Framingham Heart Study, Framingham, MA, USA.

85. Population Sciences Branch, National Heart, Lung, and Blood Institute, National Institutes of Health, Bethesda, MD.

86. Research Centre for Optimal Health, Department of Life Sciences, University of Westminster, London, W1W 6UW.

87. Departments of Psychiatry, Neurobiology \& Anatomy, Human Genetics; School of Medicine; University of Utah, Salt Lake City, UT.

88. National Institute for Health Research Imperial Biomedical Research Centre, Imperial College Healthcare NHS Trust and Imperial College London, London, UK.

89. Health Data Research-UK London substantive site, London, UK.

Corresponding authors: Gunter Schumann (gunter.schumann@kcl.ac.uk) and Paul Elliott (p.elliott@imperial.ac.uk)

\footnotetext{
ABSTRACT

Excessive alcohol consumption is one of the main causes of death and disability worldwide. Alcohol consumption is a heritable complex trait. We conducted a genome-wide association study (GWAS) of alcohol use in $~ 480,000$ people of European descent to decipher the genetic architecture of alcohol intake. We identified 46 novel, common loci, and investigated their potential functional significance using magnetic resonance imaging data, gene expression and behavioral studies in Drosophila. Our results identify new genetic pathways associated with alcohol consumption and suggest common genetic mechanisms with several neuropsychiatric disorders including schizophrenia.
} 
1 Excessive alcohol consumption is a major public health problem that is responsible

2 for $2.2 \%$ and $6.8 \%$ age-standardized deaths for women and men respectively ${ }^{1}$. Most

3 genetic studies of alcohol use focus on alcohol dependency, although the burden of

4 alcohol-related disease mainly reflects a broader range of alcohol consumption

5 behaviors in a population ${ }^{2}$. Small reductions in alcohol intake could have major public

6 health benefits; a recent study reported that even moderate daily alcohol may have

7 significant impact on mortality ${ }^{3}$.

8 Alcohol consumption is a heritable complex trait ${ }^{4}$, but genetic studies to date have

9 identified only a small number of robustly associated genetic variants ${ }^{5-8}$. These

10 include variants in the aldehyde dehydrogenase gene family, a group of enzymes that

11 catalyze the oxidation of aldehydes ${ }^{9}$, including a cluster of genes on chromosome

$124 \mathrm{q} 23(A D H 1 B, A D H 1 C, A D H 5, A D H 6, A D H 7)^{6}$.

13 Here, we report a GWAS meta-analysis of alcohol intake (g/day) among people of 14 European ancestry drawn from UK Biobank (UKB) ${ }^{10}$, the Alcohol Genome-Wide 15 Consortium (AlcGen) and the Cohorts for Heart and Aging Research in Genomic 16 Epidemiology Plus (CHARGE+) consortia. Briefly, UKB is a prospective cohort 17 study of $\sim 500,000$ individuals recruited between the ages of 40-69 years. Participants 18 were asked to report their average weekly and monthly alcohol consumption through 19 a self-completed touchscreen questionnaire ${ }^{10}$. Based on these reports, we calculated 20 the gram/day (g/d) alcohol intake (Online Methods). Participants were genotyped 21 using a customized array with imputation from the Haplotype Reference Consortium (HRC) panel $^{11}$, yielding $~ 7$ million common single nucleotide polymorphisms (SNPs) with minor allele frequency $(\mathrm{MAF}) \geq 1 \%$ and imputation quality score $[\mathrm{INFO}] \geq 0.1$. After quality control (QC) and exclusions (Online Methods) we performed GWAS of alcohol consumption using data from 404,731 UKB participants of European descent under an additive genetic model (Online Methods and Supplementary Table 1). We found that genomic inflation in the UKB analysis was $\lambda_{\mathrm{GC}}=1.45$, but did not adjust for inflation as the LD score regression intercept was 1.05, indicating that this was due to polygenicity rather than to population stratification ${ }^{12}$. The estimated SNP-wide heritability of alcohol consumption in the UKB data was 0.09 .

We also carried out GWAS in 25 independent studies from the AlcGen and CHARGE+ consortia including 76,111 participants of European descent for which alcohol g/d could be calculated (Supplementary Table 2). Various arrays were used for genotyping, with imputations performed using either the 1,000 Genomes Reference Panel or the HRC platforms (Supplementary Table 3). After QC, we applied genomic control at the individual study level and obtained summary results for $\sim 7$ million SNPs with imputation quality score $\geq 0.3$ (Online Methods). 
38 We combined the UKB, AlcGen and CHARGE+ results using a fixed effects inverse variance weighted approach for a total of 480,842 individuals ${ }^{13}$. To maximize power, we performed a single-stage analysis to test common SNPs with MAF $\geq 1 \%$. We set a stringent $P$-value threshold of $P<5 \times 10^{-9}$ to denote significance in the combined meta-analysis ${ }^{14}$, and required signals to be significant at $P<5 \times 10^{-7}$ in $\mathrm{UKB}$, with same direction of effect in UKB and AlcGen plus CHARGE+, to minimize false positive findings. We excluded SNPs within $500 \mathrm{~kb}$ of variants reported as genomewide significant in previous GWAS of alcohol consumption ${ }^{5,6}$, identified novel loci by requiring SNPs to be independent of each other ( $\left.\operatorname{LD~}^{2}<0.1\right)$, and selected the sentinel SNP within each locus according to lowest $P$-value (Online Methods).

We then tested for correlations of alcohol-associated SNPs with Magnetic Resonance Imaging (MRI) phenotypes of brain, heart and liver, and gene expression. Associations of the sentinel SNPs with other traits/diseases were investigated and Drosophila mutant models used to test for functional effects on ethanol-induced behavior.

\section{RESULTS}

Our meta-analysis identified 46 novel loci associated with alcohol consumption (log transformed g/day) (Fig. 1 and Table 1). We discovered eight additional variants in the combined analysis at nominal genome-wide significance $\left(P<1 \times 10^{-8}\right)$ that may also be associated with alcohol intake (Supplementary Table 4). The most significantly associated variant, $\operatorname{rs} 1991556\left(P<4.5 \times 10^{-23}\right)$, is an intronic variant in $M A P T$ gene that encodes the microtubule-associated protein tau, and was found through Phenoscanner not only to be associated with dementia ${ }^{15}$ and Parkinson's disease $^{16,17}$, but also with neuroticism, schizophrenia ${ }^{18}$ and other conditions ${ }^{19-21}$ encodes a member of the sine oculis homeobox transcription factor family involved in eye development ${ }^{22}$. The third SNP is $\operatorname{rs} 13107325\left(P<1.3 \times 10^{-15}\right)$, a missense SNP in SLC39A8, a gene that encodes a member of the SLC39 family of metal ion transporters, which has been associated with schizophrenia ${ }^{23}$ as well as inflammatory bowel disease, cardiovascular and metabolic phenotypes ${ }^{24-27}$ in previous GWAS (Fig. 2 and Supplementary Table 5). with SNP rs988748 $\left(P<4.4 \times 10^{-9}\right)$ in the gene encoding BDNF (brain-derived neurotrophic factor) and rs7517344, which is near ELAVL4 $\left(P=2.0 \times 10^{-10}\right)$, the gene 
product of which is involved in BDNF regulation ${ }^{28}$. Previous studies have suggested that variation in $B D N F$ is a genetic determinant of alcohol consumption and that alcohol consumption modulates BDNF expression ${ }^{29}$.

Additionally, we found association of alcohol consumption with SNP rs838145 $(P<$ $\left.3.2 \times 10^{-15}\right)$, which has been associated with macronutrient intake in a previous $\mathrm{GWAS}^{30}$. This variant is localized to IZUMO1, a locus of around $50 \mathrm{~kb}$ that spans a number of genes including FGF21, whose gene product FGF21 is a liver hormone involved in the regulation of alcohol preference, glucose and lipid metabolism ${ }^{31}$. We previously reported significant association of alcohol intake with SNP rs11940694 in $K L B$, an obligate receptor of FGF21 in the brain $^{5}$, and strongly replicated that finding here $\left(P=3.3 \times 10^{-68}\right)$.

As well as variants in $K L B$, we found support $\left(P<1 \times 10^{-5}\right)$ for association of common variants in all four of the other previously reported alcohol intake-related loci (Supplementary Table 6). These replicated loci include SNP rs6943555 in AUTS2 $\left(P=2.9 \times 10^{-6}\right)$ and variants in the alcohol dehydrogenase locus (lowest $P=$ $\left.1.2 \times 10^{-125}\right)$. In addition, we found a novel alcohol intake-related SNP rs1421085 in FTO in high $\mathrm{LD}\left(\mathrm{r}^{2}=0.92\right)$ with a variant reported previously as genome-wide significant for association with alcohol dependence ${ }^{32}$.

Conditional analysis using Genome-wide Complex Trait Analysis (GCTA) did not reveal any independent secondary signals related to alcohol consumption. Among $\sim 14,000$ individuals in the independent Airwave cohort ${ }^{33}$ (Online Methods), $7 \%$ of the variance in alcohol consumption was explained by the novel and known common variants. Using weights from our analysis, we constructed an unbiased weighted genetic risk score (GRS) in Airwave (Online Methods) and found a strong association of the novel and known variants on alcohol consumption levels $(P=2.75$ $\mathrm{x} 10^{-14}$ ), with mean difference in sex-adjusted alcohol intake of $2.6 \mathrm{~g} / \mathrm{d}$ comparing the top vs the bottom quintile of the GRS (Supplementary Table 7).

\section{Associations with MRI imaging phenotypes}

We performed single-SNP analyses of the imaging phenotypes in UKB (Online Methods) to investigate associations of our novel variants with MRI of brain $(\mathrm{N}=9,702)$, heart $(\mathrm{N}=10,706)$ and liver $(\mathrm{N}=8,479)$. With Bonferroni correction (corrected $P$-value $6.6 \times 10^{-6}$, corresponding to $0.05 / 46$ SNPs*164 imaging phenotypes), we found significant positive associations between rs13107325 and the volumes of multiple brain regions; the strongest associations were with putamen (left: 
$114 P=2.5 \times 10^{-45}$, right: $P=2.8 \times 10^{-47}$ ), ventral striatum (left: $P=9.5 \times 10^{-53}$, right: $P=$ $1159.6 \times 10^{-51}$ ) and cerebellum (strongest association for left I-IV volume; $P=1.2 \times 10^{-9}$ ) 116 (Supplementary Table 8); similar findings were also recently reported in a GWAS 117 on brain imaging in $\mathrm{UKB}^{34}$. The other significant association was for rs 1991556 with 118 the parahippocampal gyrus $\left(P=1.2 \times 10^{-6}\right)$.

119 We then tested these brain regions for association with alcohol consumption and 120 found a significant effect for the left $\left(P=2.0 \times 10^{-4}\right)$ and right $\left(P=2.6 \times 10^{-4}\right)$

121 putamen. Finally, we used data from $\mathrm{N}=8,610$ individuals and performed a mediation 122 analysis using a standard three-variable path model, bootstrapping 10,000 times to 123 calculate the significance of the mediation effect of putamen volume for genetic 124 influences on alcohol consumption (Online Methods). We found evidence that the 125 effect of SNP rs13107325 in SLC39A8 on alcohol intake is partially mediated via its 126 association with left (beta=-0.27; $P=1.9 \times 10^{-3}$ ) and right (beta=-0.26; $P=1.7 \times 10^{-3}$ ) 127 putamen volume (Fig. 3 and Supplementary Table 9).

128 We did not find any significant associations of novel SNPs with either cardiac (left 129 ventricular mass or end diastolic volume or right ventricular end diastolic volume) 130 (Supplementary Table 10) or liver fat measures on MRI (Supplementary Table 131 11), after adjustment for multiple testing.

\section{Effects of SNPs on gene expression}

133 We carried out expression quantitative trait loci eQTL analyses using the Genotype134 Tissue Expression (GTEx) and the UK Brain Expression Consortium (UKBEC) 135 datasets; 34 of the 53 novel and known SNPs associated with alcohol consumption 136 have a significant effect on gene expression in at least one tissue, including 33 SNPs 137 that affected gene expression in the brain (Supplementary Tables 12 and 13, and

138 Supplementary Fig. 1-4). We found that the most significant eQTLs often do not 139 involve the nearest gene and that several of the SNPs affect expression of different 140 genes in different tissues (Supplementary Fig. 4). For example, SNP rs 1991556 in 141 the MAPT gene affects expression of 33 genes overall, with most significant effects 142 on the expression of the non-protein coding genes CRHRI-ITI (also known as 143 C17orf69 or LINC02210) and LRRC37A4P, near MAPT, across a wide range of 144 tissues including brain, adipose tissue and skin $\left(P=7.2 \times 10^{-126}\right.$ to $\left.P=2.5 \times 10^{-6}\right)$

145 (Supplementary Fig. 4). Similarly, the A-allele at SNP rs2071305 within MYBPC3 146 affects the expression of several genes and is most significantly associated with 147 increased expression of CIQTNF4 across several tissues $\left(P=1.9 \times 10^{-25}\right.$ to $P=8.4 \mathrm{x}$ $14810^{-5}$ ). 
149 Several of these eQTLs were found to affect expression of genes known to be

150 involved in reward and addiction. SNP rs1053651 in the TCAP-PNMT-STARD3 gene

151 cluster affects expression of the PPPIRIB gene (also known as DARPP-32) which

152 encodes a protein that mediates the effects of dopamine in the mesolimbic reward

153 pathway ${ }^{35}$. Other known addiction-related genes include ANKK1 and DRD2 (affected

154 by SNP rs7121986) implicated in alcohol and nicotine dependence ${ }^{36,37}, C R H R I$

155 (affected by SNP rs1991556) involved in stress-mediated alcohol dependence ${ }^{38,39}$ and

$156 P P M 1 G$ (SNP rs1260326) whose epigenetic modification was reported to be

157 associated with alcohol abuse ${ }^{40}$.

158 Over-representation enrichment analyses based on functional annotations and disease-

159 related terms indicated that genes whose expressions are affected by the identified

160 eQTLs are most significantly enriched for terms related to abdominal cancers $(\mathrm{n}=$

161 91), motor function $(n=5)$ and cellular homeostasis $(n=22)$ (Supplementary Fig 5).

162

163

164

165

166

167

168

169

170

171

172

173

174

175

176

177

178

179

180

181

182

183

184

185

\section{Other traits and diseases}

Using LD score regression ${ }^{12}$, we assessed genetic correlations between alcohol consumption and 235 complex traits and diseases from publicly available summary GWAS statistics (Online Methods and Supplementary Table 14). The strongest positive genetic correlations based on false discovery rate $P<0.02$ were found for smoking $\left(r_{\mathrm{g}}=0.42, P=1.0 \times 10^{-23}\right)$ and HDL cholesterol levels $\left(r_{\mathrm{g}}=0.26, P=5.1 \times 10^{-}\right.$ $\left.{ }^{13}\right)$. We also found negative correlations for sleep duration $\left(\mathrm{r}_{\mathrm{g}}=-0.14, P=3.8 \times 10^{-7}\right)$ and fasting insulin levels $\left(\mathrm{r}_{\mathrm{g}}=-0.25, P=4.5 \times 10^{-6}\right)$. A significant genetic correlation was also found with schizophrenia $\left(\mathrm{r}_{\mathrm{g}}=0.07, P=3.9 \times 10^{-3}\right)$ and bipolar disorder $\left(\mathrm{r}_{\mathrm{g}}=\right.$ $0.15, P=5.0 \times 10^{-4}$ ) (Supplementary Table 14). Over-representation enrichment analysis using WebGestalt ${ }^{41}$ showed that our list of novel and known variants are significantly enriched in several diseases and traits including developmental disorder in children $\left(P<7.3 \times 10^{-5}\right)$, epilepsy $\left(P<1.4 \times 10^{-4}\right)$, heroin dependence $\left(P=5.7 \times 10^{-}\right.$ $\left.{ }^{4}\right)$ and schizophrenia $\left(P<8.4 \times 10^{-4}\right)$ (Supplementary Fig. 6). The result of Mendelian randomization analysis (Online methods) to assess a potential causal effect of alcohol on schizophrenia risk, using the inverse variance weighted approach, was not significant $(P=0.089)$, with large heterogeneity of the estimates of the tested variants.

\section{Functional studies in Drosophila}

Based on our GWAS and brain imaging findings we took forward SNP rs13107325 in SLC39A8 (alias Zip8 gene) for additional testing in Drosophila, which employ conserved mechanisms to modulate ethanol-induced behaviors ${ }^{42,43}$. First, we overexpressed human Zip8 using a Gal4-driver that included expression in neurons 
186

187

188

189

190

191

192

193

194

195

196

197

198

199

200

201

202

203

204

205

206

207

208

209

210

211

212

213

214

215

216

217

218

219

220

221

222

223

involved in multiple ethanol-induced behaviors ${ }^{43}$. Flies carrying $i c S^{\text {Gal4 }} /+U A S$ hZip 8/+ showed a slight, but significant, resistance to ethanol-induced sedation compared to control flies ( $P=0.026 ; \mathrm{N}=16$ per genotype). Ethanol tolerance, induced with repeat exposures spaced by a 4-hour recovery, was unchanged in these flies (Fig. 4a). We next used the same Gal4-driver to knock down the endogenous Drosophila ortholog of $h Z i p 8$, namely $d Z i p 71 B$. This caused the flies to display naïve sensitivity to ethanol-induced sedation, and in addition, these flies developed greater tolerance to ethanol upon repeat exposure ( $P=0.0003 ; \mathrm{N}=8$ per genotype) (Fig. $\mathbf{4 b})$. To corroborate this phenotype, we then tested flies transheterozygous for two independent transposon-insertions in the middle of the $d Z i p 71 B$ gene (Supplementary Fig. 7) and found that these $d Z i p 71 B^{M i / M B}$ flies also displayed naïve sensitivity $(P=0.006)$ and increased ethanol-induced tolerance $(P=0.032)$, compared to controls $(\mathrm{N}=8$ each) (Fig. $4 c)$.

\section{DISCUSSION}

Our discovery utilizing data on common variants from over 480,000 people of European descent has greatly extended our knowledge of the genetic architecture of alcohol intake, increasing the number of loci by nearly 10 -fold to 46 . We found loci involved in neuropsychiatric conditions such as schizophrenia, Parkinson's disease and dementia, as well as $B D N F$ where gene expression is affected by alcohol abuse. Our findings illustrate that large-scale studies of genetic associations with alcohol intake in the general population, rather than in alcohol dependency alone, can provide new insights into genetic mechanisms regulating alcohol consumption.

We highlight the role of the highly pleiotropic MAPT and SLC39A8 genes in the genetics of alcohol consumption. MAPT plays a key role in tau-associated dementia ${ }^{44}$ and both genes are also implicated in other neuropsychiatric conditions including neuroticism, schizophrenia and Parkinson's disease ${ }^{16-18}$. The SLC39A8 gene encodes a member of the SLC39 family of metal ion transporters. The encoded protein is glycosylated and found in plasma membrane and mitochondria, and is involved in the cellular transport of zinc, modulation of which could affect microglial inflammatory responses ${ }^{45}$. Our gain- and loss-of-function studies in Drosophila indicate a potential causal role of $S L C 39 A 8$ in alcohol drinking behavior. The MRI brain imaging demonstrates a significant association of SNP rs13107325 in the SLC39A8 gene and putamen volume differences, and these structural differences appear to partially mediate associations of rs 13107325 with alcohol consumption. The putamen has been associated with alcohol consumption and the withdrawal syndrome after chronic administration to rodents and non-human primates ${ }^{46}$. Putamen volume differences have also been associated with both schizophrenia and psychosis ${ }^{47,48}$ and robust 
224 association between SNP rs13107325 in SLC39A8 and schizophrenia was reported in 225 a previous GWAS $^{23}$.

226 We also report SNP rs7121986 near DRD2 as a novel alcohol intake variant in 227 GWAS. The gene product of $D R D 2$, D2 dopamine receptor, is a G protein-coupled 228 receptor on post-synaptic dopaminergic neurons that has long been implicated in 229 alcoholism $^{49}$. In addition, we identify SNP rs988748 in BDNF as a novel alcohol 230 intake variant; BDNF expression is differentially affected by alcohol exposure in 231 animal models ${ }^{50,51}$. Both genes (along with PPP1R1P) are centrally involved in 232 reward-mediating mesocortico-limbic pathways and both are implicated in the 233 development of schizophrenia. For example, there is a robust GWAS association 234 between schizophrenia and SNP rs4938021 in DRD2 (in perfect LD with our novel 235 alcohol intake-related variant rs 7121986) and DRD2 appears to be pivotal in network 236 analyses of genes involved in schizophrenia ${ }^{52}$. Taken together, our results suggest 237 that there are shared genetic mechanisms between the regulation of alcohol intake and 238 susceptibility to schizophrenia, as well as other neuropsychiatric disorders. In this 239 regard, large prospective epidemiological studies report a three-fold risk of 240 schizophrenia in relation to alcohol abuse ${ }^{53}$.

241 We previously reported genome-wide significant associations of alcohol intake with $242 K L B$, and identified a liver-brain axis linking the liver hormone FGF21 with central 243 regulation of alcohol intake involving $\beta$-Klotho receptor (the gene product of $K L B$ ) in 244 the brain ${ }^{5}$. Here, we identify a significant variant near FGF21 gene and strongly 245 replicate the previously reported $K L B$ gene variant, strengthening the genetic evidence 246 for the importance of this pathway in regulating alcohol consumption.

247 The LD score regression analysis showed a positive genetic correlation between 248 alcohol consumption, smoking and HDL cholesterol levels. This confirms previous 249 findings that reported an almost identical genetic correlation of alcohol consumption 250 with number of cigarettes per day ${ }^{54}$. Furthermore, the observed genetic correlation 251 with HDL levels is consistent with previous observations of an association between 252 alcohol consumption and $\mathrm{HDL}^{55,56}$, including results of a Mendelian randomization 253 study that suggested a possible causal role linking alcohol intake with increased HDL 254 levels $^{57}$. Finally, we found a genetic correlation (inverse) between sleep duration and 255 alcohol consumption, an association previously reported only in a few small 256 epidemiological studies ${ }^{58}$. We could not test for a genetic association between alcohol 257 and risk of alcohol-related cancers ${ }^{59}$ because of limited availability of summary data. 258 However, our gene-set enrichment analysis showed a significant enrichment for genes 259 related to abdominal cancers. 
260 Strengths of our study include its size, detailed attention to the alcohol phenotype,

261 dense coverage of the genome through imputation, incorporation of brain and other

262 imaging data to explore potential mechanisms and confirmatory Drosophila

263 functional genetic studies. Over $80 \%$ of the data came from UKB, which combines

264 high-quality phenotypic data and imputed genome-wide genetic data with strict

265 attention to quality control ${ }^{60}$. We adopted a stringent approach to claim novel variants

266 involving a conservative $P$-value threshold, internal replication in UKB and consistent

267 direction of effect with the other studies, to minimize the reporting of false positive

268 signals.

269 However, since alcohol intake is socio-culturally as well as genetically determined, it is influenced by other lifestyle and environmental factors which may modify or dilute the genetic signal. A key limitation is that assessment of alcohol intake relies on selfreport, which is prone to errors and biases including recall bias and systematic underreporting by heavy drinkers ${ }^{61,62}$. Furthermore, questionnaires on alcohol intake covered a short duration (e.g. day or week) at a single period, which may not be representative of broader drinking patterns of cohort participants. We harmonized data across cohorts by converting alcohol intake into a common metric of $\mathrm{g} / \mathrm{d}$, with imputation as necessary in UKB for participants reporting consumption of small amounts of alcohol. Taking this approach, we were able to detect strong genetic associations with alcohol intake that explained 7\% of the variance in alcohol in an independent cohort, while our GRS analysis indicates that individuals in the lower fifth of the GRS distribution were consuming daily approximately one third of a standard drink ( $2.6 \mathrm{~g} / \mathrm{d}$ alcohol) less compared with those in the upper fifth.

283

284

285

286

287

288

289

290

291

292

293

294

295

296

297

298
In summary, in this large study of genetic associations with alcohol consumption, we identified common variants in 46 novel loci with several of the genes expressed in the brain as well as other tissues. Our findings suggest that there may be common genetic mechanisms underpinning regulation of alcohol intake and development of a number of neuropsychiatric disorders including schizophrenia. This may form the basis for greater understanding of observed associations between excessive alcohol consumption and schizophrenia ${ }^{63}$.

\section{URLs}

\section{GTEx: www.gtexportal.org}

UKBEC: http://www.braineac.org/

WebGetstalt: http://www.webgestalt.org

IPA: www.qiagen.com/ingenuity

PhenoScanner: http://www.phenoscanner.medschl.cam.ac.uk (Phenoscanner

integrates results from the GWAS catalogue: https://www.ebi.ac.uk/gwas/ and

GRASP: https://grasp.nhlbi.nih.gov/) 
H.G. was funded by the NIHR Imperial College Health Care NHS Trust and Imperial College London Biomedical Research Centre. I.K. was supported by the EU PhenoMeNal project (Horizon 2020, 654241) and the UK Dementia Research Institute, which is supported by the MRC, the Alzheimer's Society and Alzheimer's Research UK. S.Thériault was supported by the Canadian Institutes of Health Research and Université Laval (Quebec City, Canada). L.R. was supported by Forschungs- und Förder-Stiftung INOVA, Vaduz, Liechtenstein. D.C. holds a McMaster University Department of Medicine Mid-Career Research Award. M.B. is supported by NIH grant R01-DK062370. P.v.d.H. was supported by ICIN-NHI and Marie Sklodowska-Curie GF (call: H2020-MSCA-IF-2014, Project ID: 661395). C.H. was supported by a core MRC grant to the MRCHGU QTL in Health and Disease research programme. N.V. was supported by Marie Sklodowska-Curie GF grant (661395) and ICIN-NHI. P.E. acknowledges support from the NIHR Biomedical 314 Research Centre at Imperial College Healthcare NHS Trust and Imperial College London, the NIHR Health Protection Research Unit in Health Impact of Environmental Hazards (HPRU-2012-10141), and the Medical Research Council (MRC) and Public Health England (PHE) Centre for Environment and Health (MR/L01341X/1). P.E. is a UK Dementia Research Institute (DRI) professor, UK DRI at Imperial College London, funded by the MRC, Alzheimer's Society and Alzheimer's Research UK. This work received support from the following sources: the European Union-funded FP6 Integrated Project IMAGEN (Reinforcement-related behaviour in normal brain function and psychopathology) (LSHM-CT- 2007-037286), the Horizon 2020 funded ERC Advanced Grant 'STRATIFY' (Brain network based stratification of reinforcement-related disorders) (695313), ERANID (Understanding the Interplay between Cultural, Biological and Subjective Factors in Drug Use Pathways) (PR-ST-0416-10004), BRIDGET (JPND: BRain Imaging, cognition Dementia and next generation GEnomics) (MR/N027558/1), the FP7 projects IMAGEMEND(602450; IMAging GEnetics for MENtal Disorders) and MATRICS (603016), the Innovative Medicine Initiative Project EU-AIMS (115300-2), the Medical Research Council Grant 'c-VEDA' (Consortium on Vulnerability to Externalizing Disorders and Addictions) (MR/N000390/1), the Swedish Research

332 Council FORMAS, the Medical Research Council, the National Institute for Health 333 Research (NIHR) Biomedical Research Centre at South London and Maudsley NHS 334 Foundation Trust and King's College London, the Bundesministeriumfür Bildung und 335 Forschung (BMBF grants 01GS08152; 01EV0711; eMED SysAlc01ZX1311A; 336 Forschungsnetz AERIAL 01EE1406A, 01EE1406B), the Deutsche 337 Forschungsgemeinschaft (DFG grants SM 80/7-2, SFB 940/2), the Medical Research 338 Foundation and Medical research council (grant MR/R00465X/1), the Human Brain 
339 Project (HBP SGA 2). Further support was provided by grants from: ANR (project 340 AF12-NEUR0008-01 - WM2NA, and ANR-12-SAMA-0004), the Fondation de 341 France, the Fondation pour la Recherche Médicale, the Mission Interministérielle de 342 Lutte-contre-les-Drogues-et-les-Conduites-Addictives (MILDECA), the Assistance343 Publique-Hôpitaux-de-Paris and INSERM (interface grant), Paris Sud University 344 IDEX 2012; the National Institutes of Health, Science Foundation Ireland 345 (16/ERCD/3797), U.S.A. (Axon, Testosterone and Mental Health during 346 Adolescence; RO1 MH085772-01A1), and by NIH Consortium grant U54 EB020403, 347 supported by a cross-NIH alliance that funds Big Data to Knowledge Centres of 348 Excellence.

\section{Conflicts/Disclosures}

B.M.P. serves on the DSMB of a clinical trial funded by the manufacturer (Zoll LifeCor) and on the Steering Committee of the Yale Open Data Access Project funded by Johnson \& Johnson.

B.W.J.H.P. has received research funding (non-related to the work reported here) from Jansen Research and Boehringer Ingelheim.

\section{Author contributions}

Central analysis: E.E., H.G., C.C., G.N., P.B., A.R.B., R.P., H.Suzuki, F.K., A.M.Y., I.K., J.E., N.D., D.L., I.T., J.D.B., P.M.M., A.R., S.D., G.S., P.E.

Writing of the manuscript: E.E., H.G., C.C., G.N., P.B., A.R.B., R.P., H.Suzuki, F.K., A.M.Y., I.K., D.L., I.T., J.D.B., P.M.M., A.R., S.D., G.S., P.E.

Association of MRI analysis: C.C., H.Suzuki, A.M.Y., A.I.B., J.D.B., P.M.M., G.S.

Alcgen and Charge+ contributor: (ARIC): A.C.M., M.R.B., B.Y., D.E.A., (CHS): B.M.P., R.N.L., T.M.B., J.A.B., (FHS): D.L., C.L., (GAPP/Swiss-AF/Beat-AF): S.Thériault, S.A., D.C., L.R., M.Kühne, (GENOA): S.L.R.K., J.A.S., W.A., S.M.R., (GRAPHIC): N.J.S., C.P.N., P.S.B., (GS): A.M.M., T-K.C., C.H., D.P., (HBCS): L.J., S.Tuominen, M.M.P., J.G.E., (HRS): D.R.W., S.L.R.K., J.D.F., W.Z., J.A.S., (MESA): X.G., J.Y., A.W., J.I.R., (METSIM): M.L., A.S., J.Vangipurapu, J.K., (FUSION): M.B., K.L.M., L.J.S., A.U.J., (NESDA): B.W.J.H.P., Y.M., (NFBC): MR.J., J.Veijola, M.Männikkö, J.A., (ORCADES): H.C., P.K.J, (VIKING): J.F.W., K.A.K., (Croatia-VIS): I.R., O.P., (Croatia-KORCULA): C.H., (PREVEND): N.V., P.v.d.H, (OZALC): M.G.N., J.B.W., P.A.L., A.C.H., (SHIP): A.T., H.J.G., S.E.B., G.H., (TRAILS-pop): A.J.O, I.M.N., (TRAILS-CC): C.A.H., H.Snieder , (TwinsUK): 


\section{References}

385

1. GBD 2016 Alcohol Collaborators. Alcohol use and burden for 195 countries and territories, 1990-2016: a systematic analysis for the Global Burden of Disease Study 2016. Lancet (2018).

2. World Health Organization. Global status report on alcohol and health 2018. (2018).

3. Wood, A.M. et al. Risk thresholds for alcohol consumption: combined analysis of individual-participant data for 599912 current drinkers in 83 prospective studies. Lancet 391, 1513-1523 (2018).

4. Verhulst, B., Neale, M.C. \& Kendler, K.S. The heritability of alcohol use disorders: a meta-analysis of twin and adoption studies. Psychol Med 45, 1061-72 (2015).

5. Schumann, G. et al. KLB is associated with alcohol drinking, and its gene product beta-Klotho is necessary for FGF21 regulation of alcohol preference. Proc Natl Acad Sci U S A 113, 14372-14377 (2016).

6. Clarke, T.K. et al. Genome-wide association study of alcohol consumption and genetic overlap with other health-related traits in UK Biobank $(\mathrm{N}=112$ 117). Mol Psychiatry 22, 1376-1384 (2017).

7. Jorgenson, E. et al. Genetic contributors to variation in alcohol consumption vary by race/ethnicity in a large multi-ethnic genome-wide association study. Mol Psychiatry 22, 1359-1367 (2017).

8. Baik, I., Cho, N.H., Kim, S.H., Han, B.G. \& Shin, C. Genome-wide association studies identify genetic loci related to alcohol consumption in Korean men. Am J Clin Nutr 93, 809-16 (2011).

9. Jackson, B. et al. Update on the aldehyde dehydrogenase gene (ALDH) superfamily. Hum Genomics 5, 283-303 (2011).

10. Sudlow, C. et al. UK biobank: an open access resource for identifying the causes of a wide range of complex diseases of middle and old age. PLoS Med 12, e1001779 (2015).

11. McCarthy, S. et al. A reference panel of 64,976 haplotypes for genotype imputation. Nat Genet 48, 1279-83 (2016).

12. Bulik-Sullivan, B.K. et al. LD Score regression distinguishes confounding from polygenicity in genome-wide association studies. Nat Genet 47, 291-5 (2015).

13. Evangelou, E. \& Ioannidis, J.P. Meta-analysis methods for genome-wide association studies and beyond. Nat Rev Genet 14, 379-89 (2013).

14. Evangelou, E. et al. Genetic analysis of over 1 million people identifies 535 new loci associated with blood pressure traits. Nat Genet 50, 1412-1425 (2018).

15. Desikan, R.S. et al. Genetic overlap between Alzheimer's disease and Parkinson's disease at the MAPT locus. Mol Psychiatry 20, 1588-95 (2015). 
16. Do, C.B. et al. Web-based genome-wide association study identifies two novel loci and a substantial genetic component for Parkinson's disease. PLoS Genet 7, e1002141 (2011).

17. Pankratz, N. et al. Meta-analysis of Parkinson's disease: identification of a novel locus, RIT2. Ann Neurol 71, 370-84 (2012).

18. Okbay, A. et al. Genetic variants associated with subjective well-being, depressive symptoms, and neuroticism identified through genome-wide analyses. Nat Genet 48, 624-33 (2016).

19. Couch, F.J. et al. Genome-wide association study in BRCA1 mutation carriers identifies novel loci associated with breast and ovarian cancer risk. PLoS Genet 9, e1003212 (2013).

20. Ikram, M.A. et al. Common variants at $6 \mathrm{q} 22$ and $17 \mathrm{q} 21$ are associated with intracranial volume. Nat Genet 44, 539-44 (2012).

21. van der Harst, P. et al. Seventy-five genetic loci influencing the human red blood cell. Nature 492, 369-75 (2012).

22. Samuel, A. et al. Six3 regulates optic nerve development via multiple mechanisms. Sci Rep 6, 20267 (2016).

23. Schizophrenia Working Group of the Psychiatric Genomics, C. Biological insights from 108 schizophrenia-associated genetic loci. Nature 511, 421-7 (2014).

24. Liu, J.Z. et al. Association analyses identify 38 susceptibility loci for inflammatory bowel disease and highlight shared genetic risk across populations. Nat Genet 47, 979-986 (2015).

25. International Consortium for Blood Pressure Genome-Wide Association Studies et al. Genetic variants in novel pathways influence blood pressure and cardiovascular disease risk. Nature 478, 103-9 (2011).

26. Speliotes, E.K. et al. Association analyses of 249,796 individuals reveal 18 new loci associated with body mass index. Nat Genet 42, 937-48 (2010).

27. Teslovich, T.M. et al. Biological, clinical and population relevance of 95 loci for blood lipids. Nature 466, 707-13 (2010).

28. Lim, C.S. \& Alkon, D.L. Protein kinase C stimulates HuD-mediated mRNA stability and protein expression of neurotrophic factors and enhances dendritic maturation of hippocampal neurons in culture. Hippocampus 22, 2303-19 (2012).

29. Barker, J.M., Taylor, J.R., De Vries, T.J. \& Peters, J. Brain-derived neurotrophic factor and addiction: Pathological versus therapeutic effects on drug seeking. Brain Res 1628, 68-81 (2015).

30. Tanaka, T. et al. Genome-wide meta-analysis of observational studies shows common genetic variants associated with macronutrient intake. Am J Clin Nutr 97, 1395-402 (2013).

31. Talukdar, S. et al. FGF21 Regulates Sweet and Alcohol Preference. Cell Metab 23, 344-9 (2016).

32. Grant, S.F. et al. Association analysis of the FTO gene with obesity in children of Caucasian and African ancestry reveals a common tagging SNP. PLoS One 3, e1746 (2008). 
33. Elliott, P. et al. The Airwave Health Monitoring Study of police officers and staff in Great Britain: rationale, design and methods. Environ Res 134, 280-5 (2014).

34. Elliott, L.T. et al. Genome-wide association studies of brain imaging phenotypes in UK Biobank. Nature 562, 210-216 (2018).

35. Stipanovich, A. et al. A phosphatase cascade by which rewarding stimuli control nucleosomal response. Nature 453, 879-84 (2008).

36. Yang, B.Z. et al. Association of haplotypic variants in DRD2, ANKK1, TTC12 and NCAM1 to alcohol dependence in independent case control and family samples. Hum Mol Genet 16, 2844-53 (2007).

37. Gelernter, J. et al. Haplotype spanning TTC12 and ANKK1, flanked by the DRD2 and NCAM1 loci, is strongly associated to nicotine dependence in two distinct American populations. Hum Mol Genet 15, 3498-507 (2006).

38. Treutlein, J. et al. Genetic association of the human corticotropin releasing hormone receptor 1 (CRHR1) with binge drinking and alcohol intake patterns in two independent samples. Mol Psychiatry 11, 594-602 (2006).

39. Timpl, P. et al. Impaired stress response and reduced anxiety in mice lacking a functional corticotropin-releasing hormone receptor 1. Nat Genet 19, 162-6 (1998).

40. Ruggeri, B. et al. Association of Protein Phosphatase PPM1G With Alcohol Use Disorder and Brain Activity During Behavioral Control in a GenomeWide Methylation Analysis. Am J Psychiatry 172, 543-52 (2015).

41. Wang, J., Vasaikar, S., Shi, Z., Greer, M. \& Zhang, B. WebGestalt 2017: a more comprehensive, powerful, flexible and interactive gene set enrichment analysis toolkit. Nucleic Acids Res 45, W130-W137 (2017).

42. Gonzalez, D.A. et al. The Arf6 activator Efa6/PSD3 confers regional specificity and modulates ethanol consumption in Drosophila and humans. Mol Psychiatry 23, 621-628 (2018).

43. Ojelade, S.A. et al. Rsu1 regulates ethanol consumption in Drosophila and humans. Proc Natl Acad Sci U S A 112, E4085-93 (2015).

44. Rademakers, R., Cruts, M. \& van Broeckhoven, C. The role of tau (MAPT) in frontotemporal dementia and related tauopathies. Hum Mutat 24, 277-95 (2004).

45. Higashi, Y. et al. Influence of extracellular zinc on M1 microglial activation. Sci Rep 7, 43778 (2017).

46. Chen, G. et al. Striatal involvement in human alcoholism and alcohol consumption, and withdrawal in animal models. Alcohol Clin Exp Res 35, 1739-48 (2011).

47. Okada, N. et al. Abnormal asymmetries in subcortical brain volume in schizophrenia. Mol Psychiatry 21, 1460-6 (2016).

48. van Erp, T.G. et al. Subcortical brain volume abnormalities in 2028 individuals with schizophrenia and 2540 healthy controls via the ENIGMA consortium. Mol Psychiatry 21, 547-53 (2016).

49. Meyers, J.L. et al. The association between DRD2/ANKK1 and genetically informed measures of alcohol use and problems. Addict Biol 18, 523-36 (2013). 
50. Logrip, M.L., Barak, S., Warnault, V. \& Ron, D. Corticostriatal BDNF and alcohol addiction. Brain Res 1628, 60-7 (2015).

51. Boschen, K.E., Criss, K.J., Palamarchouk, V., Roth, T.L. \& Klintsova, A.Y. Effects of developmental alcohol exposure vs. intubation stress on BDNF and TrkB expression in the hippocampus and frontal cortex of neonatal rats. Int $J$ Dev Neurosci 43, 16-24 (2015).

52. Monaco, A. et al. A complex network approach reveals a pivotal substructure of genes linked to schizophrenia. PLoS One 13, e0190110 (2018).

53. Nielsen, S.M., Toftdahl, N.G., Nordentoft, M. \& Hjorthoj, C. Association between alcohol, cannabis, and other illicit substance abuse and risk of developing schizophrenia: a nationwide population based register study. Psychol Med 47, 1668-1677 (2017).

54. Nivard, M.G. et al. Connecting the dots, genome-wide association studies in substance use. Mol Psychiatry 21, 733-5 (2016).

55. Gaziano, J.M. et al. Moderate alcohol intake, increased levels of high-density lipoprotein and its subfractions, and decreased risk of myocardial infarction. $N$ Engl J Med 329, 1829-34 (1993).

56. Linn, S. et al. High-density lipoprotein cholesterol and alcohol consumption in US white and black adults: data from NHANES II. Am J Public Health 83, 811-6 (1993).

57. $\mathrm{Vu}, \mathrm{K} . \mathrm{N}$. et al. Causal Role of Alcohol Consumption in an Improved Lipid Profile: The Atherosclerosis Risk in Communities (ARIC) Study. PLoS One 11, e0148765 (2016).

58. Chaput, J.P., McNeil, J., Despres, J.P., Bouchard, C. \& Tremblay, A. Short sleep duration is associated with greater alcohol consumption in adults. Appetite 59, 650-5 (2012).

59. Bagnardi, V. et al. Alcohol consumption and site-specific cancer risk: a comprehensive dose-response meta-analysis. Br J Cancer 112, 580-93 (2015).

60. Bycroft, C. et al. The UK Biobank resource with deep phenotyping and genomic data. Nature 562, 203-209 (2018).

61. Boniface, S., Kneale, J. \& Shelton, N. Drinking pattern is more strongly associated with under-reporting of alcohol consumption than sociodemographic factors: evidence from a mixed-methods study. BMC Public Health 14, 1297 (2014).

62. Greenfield, T.K. \& Kerr, W.C. Alcohol measurement methodology in epidemiology: recent advances and opportunities. Addiction 103, 1082-99 (2008).

63. Hambrecht, M. \& Hafner, H. Substance abuse and the onset of schizophrenia. Biol Psychiatry 40, 1155-63 (1996). 
Figure 1. Manhattan plot showing $P$-values from discovery genome-wide association meta-analysis with alcohol intake (g/d) among 480,842 individuals across UK Biobank, AlcGen and CHARGE+, excluding known variants. The $P$ -

561 value was computed using inverse variance fixed effects models. The y axis shows the

562 $-\log _{10} P$ values and the $\mathrm{x}$ axis shows their chromosomal positions. Horizontal blue

563 line represents the threshold of $P=5 \times 10^{-9}$.

564

565

566

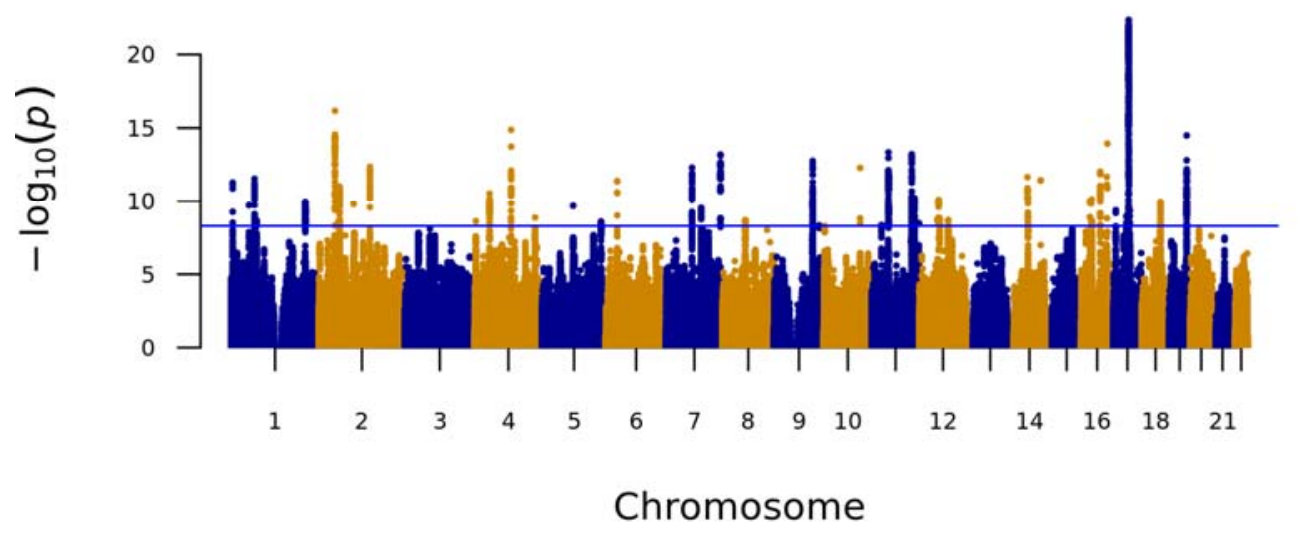


568 Figure 2. Association of alcohol intake loci with other traits. Plot shows results 569 from associations with other traits which were extracted from the PhenoScanner 570 database for the 46 novel sentinel SNPs including proxies in Linkage Disequilibrium $571 \quad\left(r^{2} \geq 0.8\right)$ with genome-wide significant associations.

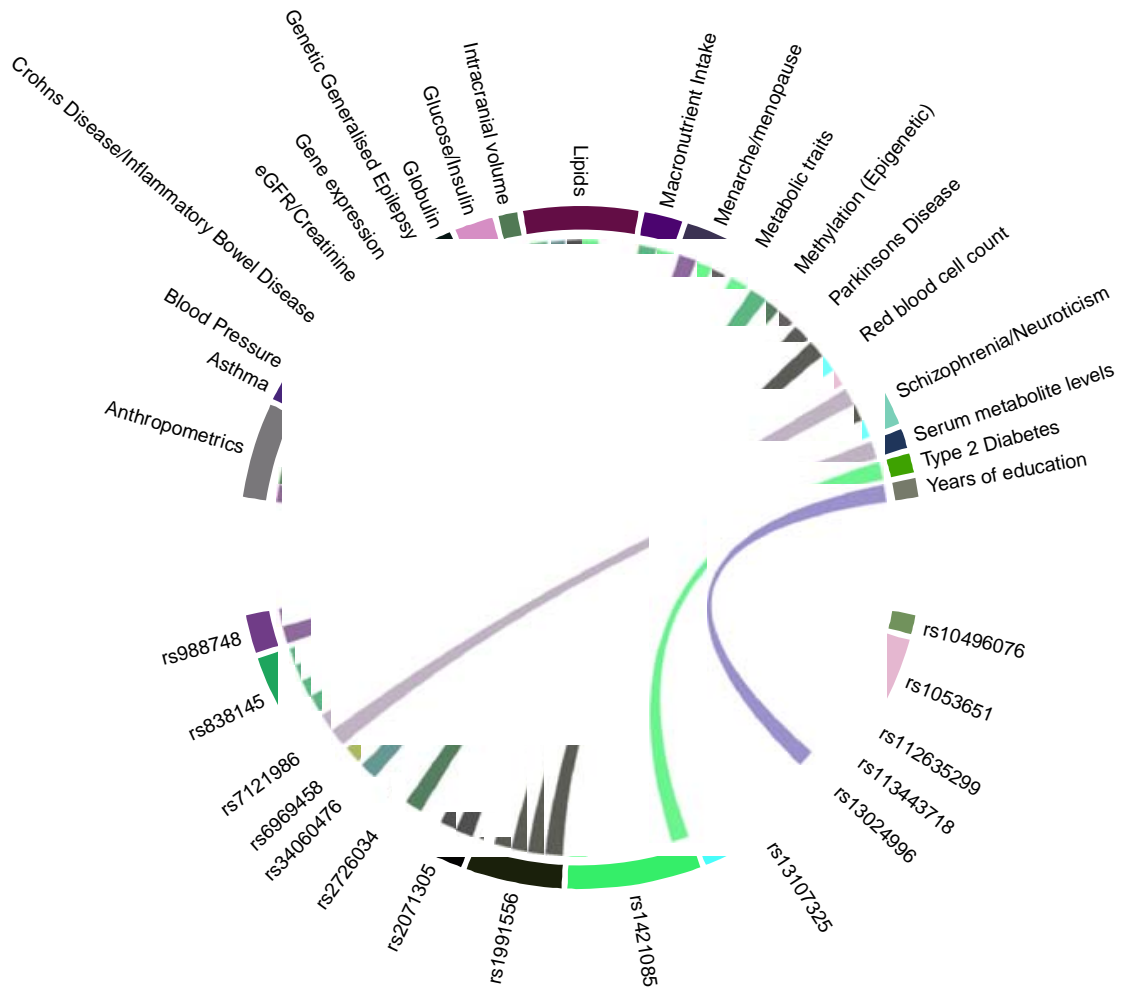


Figure 3. Mediation effect of bilateral putamen on the relationship between SNP rs13107325 and alcohol intake. Left putamen is indicated by the green color whereas the right putamen by the red. a presents the association between rs 13107325 and putamen, $\mathrm{b}$ is the association between putamen and alcohol consumption, $\mathrm{c}$ the association of rs13107325 and alcohol consumption, c' is the association between rs13107325 and alcohol consumption after excluding the effect of putamen, and ab is the mediation effect. The significance of the effect is based on bootstrapping. We provide the z-statistic for each relationship combined with $P$-values (** as $P<0.005$, * as $P<0.1$ ).

584

585

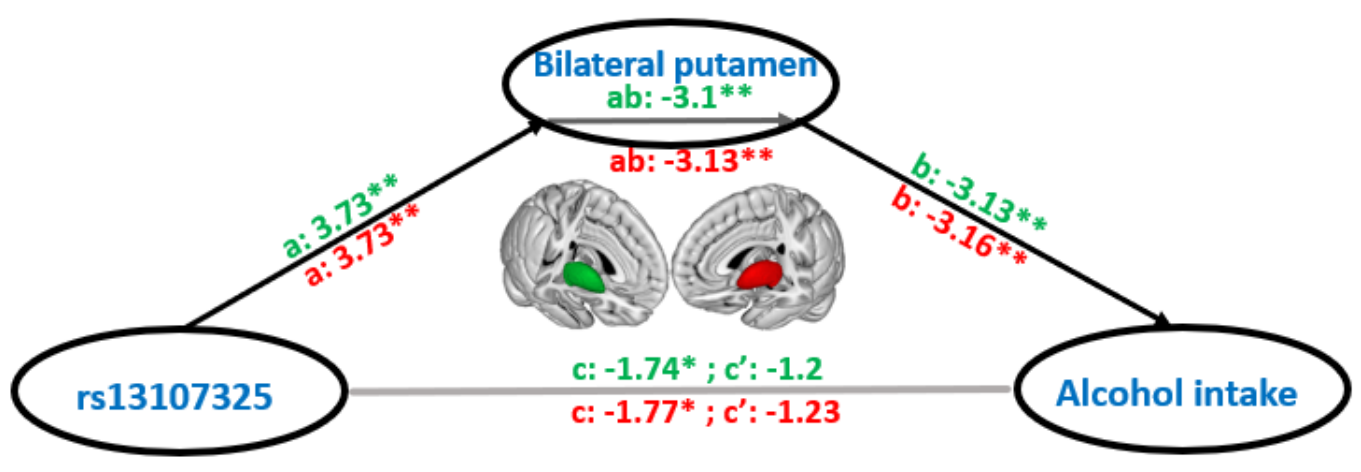


593 Figure 4. Comparison of Zip8 alcohol phenotypes in Drosophila. Flies were

594 exposed to 100/50 Ethanol/Air vapor for $30 \mathrm{~min}$ for exposure 1, and the time to $50 \%$

595 loss of righting was determined (ST-50, sedation time). After recovery on food for 4

596 hours, flies were re-exposed to the same vapors, and the second ST-50 recorded (left

597 side). The resulting increase in ST-50, i.e. tolerance, is shown on the right. In a)

598 overexpressed human hZIP8 in ics-expressing cells flies are compared against

599 controls whereas in b) knockdown of the fly ortholog $d$ Zip $71 B$ is compared against

600 controls. In c) flies carrying two transposon insertions in the endogenous $d$ Zip $71 B$

601 gene are compared against controls. Significance levels: $* * * P<0.001, * * P<0.01$,

$602 * * P<0.05$. Actual $P$-values are presented in the text

a

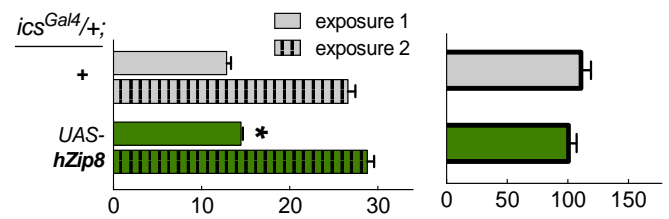

b

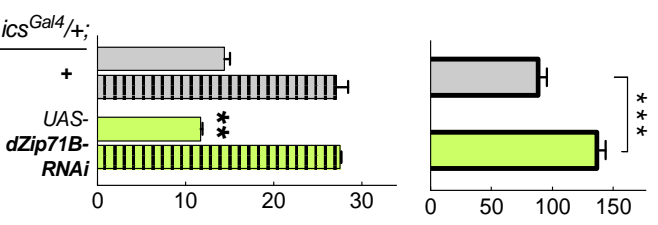

C

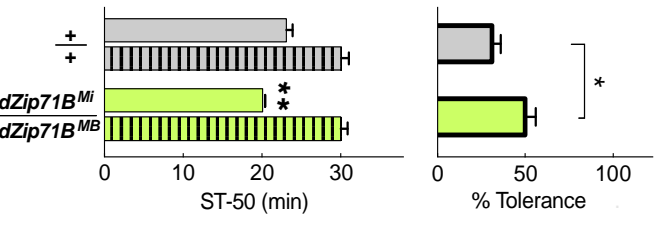

603

604 
605

606

607

608

609

610

611

612

613

614

615

616

617

618

619

620

621

622

623

624

625

626

627

628

629

630

631

632

633

634

635

636

637

638

639

640

641

642

643

644

\section{ONLINE METHODS}

\section{UK Biobank data}

We conducted a Genome Wide Association Study (GWAS) analysis among 458,577 UKB participants of European descent, identified from a combination of self-reported and genetic data. The details of the selection of the participants has been described elsewhere ${ }^{14}$. These comprise 408,951 individuals from UKB genotyped at 825,927 variants with a custom Affymetrix UK Biobank Axiom Array chip and 49,626 individuals genotyped at 807,411 variants with a custom Affymetrix UK BiLEVE Axiom Array chip from the UK BiLEVE study, which is a subset of UKB. For our analyses, we used SNPs imputed centrally by UKB using the Haplotype Reference Consortium (HRC) panel.

\section{Alcohol intake}

We calculated the alcohol intake as grams of alcohol per day (g/d) based on selfreported alcohol drinking from the touch-screen questionnaire. The quantity of each type of drink (red wine, white wine, beer/cider, fortified wine, spirits) was multiplied by its standard drink size and reference alcohol content. Drink-specific intake during the reported drinking period (a week for frequent drinkers defined as: daily or almost daily/once or twice a week/three or four times a week; or a month for occasional drinkers defined as: one to three times a month/special occasions only) was summed up and converted to $\mathrm{g} / \mathrm{d}$ alcohol intake for all participants with complete response to the quantitative drinking questions. The alcohol intake for participants with incomplete response was imputed by bootstrap resampling from the complete responses, stratified by drinking frequency (occasional or frequent) and sex.

Participants were defined as life-time non-drinkers if they reported 'never' on the question on alcohol drinking frequency (UKB field 1558) and 'no' for the question on former drinker (UKB field 3731); they were excluded from further analysis. Participants with daily alcohol consumption > 500 grams we considered outliers and they were dropped from the analyses. We also excluded participants with missing covariates, leaving data on 404,732 individuals. We $\log _{10}$ transformed $\mathrm{g} / \mathrm{d}$ alcohol and sex-specific residuals were derived from the regression of $\log _{10}$ transformed $\mathrm{g} / \mathrm{d}$ alcohol on age, age ${ }^{2}$, genotyping chip and weight.

\section{UKB genetic analysis}

We performed linear mixed modeling using BOLT-LMM software ${ }^{64}$, under an additive genetic model, for associations of measured and imputed SNPs with alcohol consumption (sex-specific residuals of the $\log _{10}$ transformed $\mathrm{g} / \mathrm{d}$ variable). Model building was based on SNPs with MAF $>5 \%$, call rate $>98.5 \%$ and HWE P > $1 \times 10^{-}$ 
$645{ }^{6}$. SNPs were imputed using the HRC panel with imputation quality INFO score $>0.1$. 646 We estimated the LD score regression (LDSR) intercept to access the degree of 647 genomic inflation beyond polygenicity as well as the lambda inflation factor $\lambda_{\mathrm{GC}}{ }^{65}$.

648 The Alcohol Genome-Wide Consortium (AlcGen) and the Cohorts for Heart and 649 Aging Research in Genomic Epidemiology Plus (CHARGE+) consortia

650 We analyzed available GWAS data from 25 independent studies $(\mathrm{N}=76,111)$ from the 651 AlcGen and the CHARGE+ consortia. All study participants were of reported 652 European ancestry and data were imputed to either the 1000 Genome Project or the 653 HRC panel. Alcohol intake in $\mathrm{g} / \mathrm{d}$ was computed and the $\log _{10}$ transformed residuals 654 were analyzed as described above. Study names, cohort information and general study 655 methods are included in Supplementary Table 2 and 3.

656 All studies were centrally quality-controlled using easyQC ${ }^{66}$. Finally, we analyzed 657 data on $~ 7.1$ M SNPs at MAF $>1 \%$ and imputation quality score (Impute [Info score] 658 or Mach $\left.\left[\mathrm{r}^{2}\right]\right)>0.3$. Genomic control (GC) was applied at study level. We synthesized 659 the available GWAS using a fixed effects inverse variance weighted meta-analysis 660 and summary estimates were derived for AlcGen and CHARGE+.

\section{One-stage meta-analysis}

662 We performed a one-stage meta-analysis applying a fixed-effects inverse variance 663 weighted meta-analysis using METAL ${ }^{67}$ to obtain summary results from the UKB and 664 and the AlcGen plus CHARGE+ GWAS, for up to $\mathrm{N}=480,842$ participants and $~ 7.1$ 665 M SNPs with MAF $\geq 1 \%$ for variants present in both the UKB data and AlcGen and 666 CHARGE+ meta-analysis. The LDSR intercept (standard error), in the discovery 667 meta-analysis was 1.05 and no further correction was applied.

\section{Previously reported (known) SNPs}

670 We looked up in the GWAS catalog (http://www.ebi.ac.uk/gwas/) and identified 17

671 SNPs that associated with alcohol consumption at genome-wide significance level $(P$ $672<5 \times 10^{-8}$ ). We enhanced the list by reference to a recent GWAS by Clarke et $\mathrm{al}^{6}$ that 673 was not covered by the GWAS catalog at the time of the analysis, reporting 14 674 additional rare and common novel SNPs. Together with a SNP in RASGRF2 shown to 675 be associated with alcohol-induced reinforcement ${ }^{68}$, we found 31 previously reported 676 alcohol consumption related SNPs.

\section{Novel loci}

679 According to locus definition of i) SNPs within $\pm 500 \mathrm{~kb}$ distance of each other; ii) 680 SNPs in linkage disequilibrium LD $\left(\mathrm{r}^{2}>0.1\right)$ calculated with PLINK, we augmented 681 the list of known SNPs to all SNPs present within our data, not contained within the 
682 previously published loci. We further excluded SNPs in the HLA region 683 (chromosome 6, 25-34Mb) due to its complex LD structure. We performed LD 684 clumping in PLINK on 4,515 unknown SNPs with $P<1 \times 10^{-8}$ using an $r^{2}>0.1$ and 685 distance threshold of $500 \mathrm{~kb}$. We further grouped the lead SNPs within $500 \mathrm{~kb}$ from each other into the same loci and selected the SNP with smallest $P$-value from the locus as sentinel SNP.

688

To report a SNP as novel signal of association with alcohol consumption:

689

690

691

692

i) the sentinel SNP has $P<5 \times 10^{-9}$ in the one-stage meta-analysis;

ii) the sentinel SNP is strongly associated $\left(P<5 \times 10^{-7}\right)$ in the UKB GWAS

693

694 alone;

iii) the sentinel SNP has concordant direction of effect between UKB and AlcGen and CHARGE+ datasets;

iv) The sentinel SNP is not located within any of the previously reported loci

695

696

697

698

699

700

701

702

703

704

705

706

707

708

709

710

711

712

713

714

715

716

717

718

719

We selected the above criteria i) to iii) to minimize false positive findings including use of a conservative one-stage $P$-value threshold that is an order of magnitude more stringent than a genome-wide significance $P$-value. (The threshold of $P<5 \times 10^{-9}$ has been proposed e.g. for whole-genome sequencing-based studies.) This approach led us to the identification of 46 sentinel SNPs in total.

\section{Conditional analysis}

We conducted locus-specific conditional analysis using the GCTA (Genome-wide Complex Trait Analysis) software (http://cnsgenomics.com/software/gcta). For each of the 46 novel sentinel SNPs, we obtained conditional analysis results for the SNPs with MAF> $1 \%$ and within $500 \mathrm{~kb}$ from the sentinel SNP after conditioning on the sentinel SNP. The meta-analysis results of the GWAS in UKB, AlcGen and CHARGE+ were used as input summary statistics and the individual-level genetic data from UKB were used as the reference sample. Results for a SNP were considered conditionally significant if the difference between the conditional $P$-value and the original $P$-value is greater than 1.5 -fold $\left(-\log _{10} P /-\log _{10}\left(P \_\right.\right.$conditional $)>1.5$ and the conditional $P$-value is smaller than $5 \times 10^{-8}$.

\section{Gene expression analyses}

To analyze the impact of genetic variants on expression of neighboring genes and identify expression quantitative trait loci (cis-eQTLs; i.e., SNPs associated with differences in local gene expression), we used two publicly available databases, the Genotype-Tissue Expression (GTEx) database ${ }^{69}$ and the UK Brain Expression Consortium (UKBEC) dataset ${ }^{70}$. We searched these databases for significant varianttranscripts pairs for genes within $1 \mathrm{Mb}$ of each input SNP. 
720 With the GTEx database, we tested for cis-eQTL effects in 48 tissues from 620

721 donors. The data described herein were obtained from the GTEx Portal, Release: V7 722 and used FastQTL ${ }^{71}$, to map SNPs to gene-level expression data and calculate q723 values based on beta distribution-adjusted empirical $P$-values ${ }^{72}$. A false discovery rate 724 (FDR) threshold of $\leq 0.05$ was applied to identify genes with a significant eQTL. The 725 effect size, defined as the slope of the linear regression, was computed in a 726 normalized space (normalized effect size (NES)), where magnitude has no direct 727 biological interpretation. Here, NES reflects the effects of our GWAS A1 alleles (that 728 are not necessarily the alternative alleles relative to the reference alleles, as reported 729 in the GTEx database). Supplementary Table 12 lists transcripts-SNPs associations 730 with significant eQTL effects.

731 With the UKBEC dataset that comprises 134 brains (http://www.braineac.org/), we 732 searched for cis-eQTLs in 10 brain regions, including the cerebellar cortex (CRBL), 733 frontal cortex (FCTX), hippocampus (HIPP), medulla (specifically inferior olivary 734 nucleus, MEDU), occipital cortex (specifically primary visual cortex, OCTX), 735 putamen (PUTM), substantia nigra (SNIG), thalamus (THAL), temporal cortex 736 (TCTX) and intralobular white matter (WHMT), as well as across all brain tissues 737 (aveALL). MatrixEQTL ${ }^{73}$ generated $P$-values for each expression profile (either 738 exon-level or gene-level) against the respective SNP were obtained for the 10 739 different tissues and overall (aveALL). Supplementary Table 13 lists transcripts740 SNPs associations with a eQTL $P$-value $<0.0045$ in at least one brain tissue. 741 Subsequent data analysis was performed in R (http://www.R-project.org/).

742 We carried out over-representation enrichment analysis using the list of 146 GTEx 743 eQTL genes. Ingenuity pathway analysis (IPA®, QIAGEN Inc.) was performed on 744 this list using ontology annotations from all available databases except those derived 745 from low-confidence computational predictions.

747 Magnetic Resonance Imaging Data

748 We used the most recent release of magnetic resonance imaging (MRI) data on brain, 749 heart and liver for UKB participants to investigate genetic associations with the 46 750 novel SNPs for alcohol consumption.

\section{Brain imaging}

\section{Brain MRI acquisition and pre-processing}

We used the T1 data from UKB to elucidate volumetric brain structures, including the cortical and the sub-cortical areas. The $\mathrm{T} 1$ data were acquired and pre-processed centrally by UKB. The brain regions were defined by combining the Harvard-Oxford 
758 cortical and subcortical atlases ${ }^{74}$ (https://fsl.fmrib.ox.ac.uk/fsl/fslwiki/Atlases) and the 759 Diedrichsen cerebellar atlas ${ }^{75}$ (http://www.diedrichsenlab.org/imaging/propatlas.htm). 760 FAST (FMRIB's Automated Segmentation Tool ${ }^{76}$ was then used to estimate the grey 761 matter partial volume within each brain region. Subcortical region volumes were also 762 modelled by using FIRST (FMRIB's Integrated Registration and Segmentation Tool). 763 More details about the MRI scanning protocol and pre-processing has been provided

764 in UKB documentation (https://biobank.ctsu.ox.ac.uk/crystal/docs/brain_mri.pdf).

765

766

767

768

769

770

771

772

773

774

775

776

777

778

779

780

781

782

783

784

785

786

787

788

789

790

791

792

793

794

795

796

\section{Association Analyses}

We performed association analyses on $\mathrm{N}=9,705$ individuals between all novel SNPs and the grey matter volume of brain regions using Pearson correlation, adjusting for age, age ${ }^{2}$, sex, age $\times$ sex, $\operatorname{age}^{2} \times$ sex, and head size. All, brain volume features, log transformed alcohol intake data $(\mathrm{g} / \mathrm{d})$, and the confounders were firstly transformed by using a rank-based inverse Gaussian transformation. Significance levels were set at $P$ $<0.05$ adjusted using the false-discovery rate method for multiple comparisons.

\section{Mediation analysis}

To assess if the effect of a SNP on alcohol consumption is mediated through a brain region, we performed a single-level mediation analysis based on a standard threevariable path model (SNP-brain region-alcohol consumption) with corrected and accelerated percentile bootstrapping 10,000 times to calculate the significance of the mediation effect. We considered as mediator variable the grey matter volume of brain regions that had a significant association on alcohol consumption. We calculated the significance of path $a$, path $b$ and $a * b$ mediation (SNP-brain region-alcohol consumption) using a multilevel mediation and moderation (M3) toolbox ${ }^{77,78}$

\section{Cardiac Imaging}

\section{Cardiac MRI acquisition and pre-processing}

Details of the cardiac image acquisition in UKB are reported previously ${ }^{79}$. Cardiac MRI was acquired using a clinical wide bore 1.5T scanner (MAGNETOM Aera, Syngo Platform VD13A, Siemens Healthcare, Erlangen, Germany) with 48 receiver channels, a $45 \mathrm{mT} / \mathrm{m}$ and $200 \mathrm{~T} / \mathrm{m} / \mathrm{s}$ gradient system, an 18-channel anterior body surface coil used in combination with 12 elements of an integrated 32 element spine coil and electrocardiogram gating for cardiac synchronization. A two-dimensional short-axis cardiac MRI was obtained using a balanced steady state free precession to cover the entire left and right ventricle (echo time, $1.10 \mathrm{msec}$; repetition time, $2.6 \mathrm{msec}$; flip angle, $80^{\circ}$; slice thickness, $8 \mathrm{~mm}$ with $2 \mathrm{~mm}$ gap; typical field of view, $380 \times 252 \mathrm{~mm}$; matrix size, $208 \times 187$, acquisition of 1 slice per breath-hold). 
797 The cardiac images were segmented to provide left ventricular mass (LVM), left end-

798 diastolic (LVEDV), left end-systolic volume (LVESV), and right end-diastolic 799 (RVEDV) and right end-systolic volume (RVESV) using a fully convolutional 800 network as described previously ${ }^{80}$. Left (LVEF) and right ventricular ejection fraction 801 (RVEF) were derived from (LVEDV-LVESV)/LVEDV $\times 100$ and (RVEDV802 RVESV)/RVEDV×100, respectively.

803

804

805

806

807

808

809

810

811

812

813

814

815

816

817

818

819

820

821

822

823

824

825

826

827

828

829

830

831

832

833

834

835

836

\section{Association Analyses}

To test associations between cardiac MRI measures and alcohol consumption-related SNPs, we carried out a regression of LVM, LVEDV, LVEF, RVEDV, and RVEF onto each of the 46 SNPs adjusting for age, sex, height, weight, hypertension, diabetes, and smoking history. Significance levels were set at $P<0.05$ adjusted using the false-discovery rate method for multiple comparisons.

\section{Liver Imaging}

Liver MRI acquisition and pre-processing

Details of the liver image acquisition protocol have been reported previously ${ }^{81}$. Briefly, all participants were scanned in a Siemens MAGNETOM Aera 1.5-T MRI scanner (Siemens Healthineers, Erlangen, Germany) using a 6-minute dual-echo Dixon Vibe protocol, providing a water and fat separated volumetric data set for fat and muscle covering neck to knees. For liver proton density fat fraction (PDFF) quantification, an additional single multi-echo gradient slice was acquired over the liver. Liver images were analysed by computing specific ROI for water, fat and $\mathrm{T} 2 *$ by magnitude-based chemical shift technique with a 6-peak lipid model, correcting for $\mathrm{T} 1$ and $\mathrm{T} 2 *$

\section{Association Analyses}

We performed association analyses between 46 alcohol consumption-related SNPs and liver PDFF (\%), from 8,372 samples, using a linear regression model adjusting for age, age ${ }^{2}$, sex, T2D, BMI, genotyping chip and first three PCs. Liver PDDF was firstly transformed by using a rank-based inverse transformation. Significance levels were set at $P<0.05$ adjusted using the false-discovery rate method for multiple comparisons.

\section{Drosophila experiments}

Flies were kept on standard cornmeal/molasses fly food in a 12:12hr light:dark cycle at $25^{\circ} \mathrm{C}$. Transgenc flies were obtained from the Bloomington Drosophila Stock Center: UAS-hZip8 BL\#66125, UAS-dZIP71B-TRiP-RNAi ${ }^{H M C 04064}$ BL\#55376, 
$837 d Z i p 71 B^{M 113940}$ BL\#59234, and $d Z i p 71 B^{M B 11703}$ BL\#29928. For behavioral 838 experiments, crosses were set up such that experimental and control flies were sibling 839 progeny from a cross, and both were therefore in the same hybrid genetic background ( $w$ Berlin / unknown). Flies aged 1-5 days of adult age were collected, exposed to

841

842

843

844

845

846

847

848

849

850

851

852

853

854

855

856

857

858

859

860

861

862

863

864

865

866

867

868

869

870

871

872

873

874 100/50 (flowrates) ethanol/air vapor in the Booze-o-Mat 2 days later, and their loss of righting determined by slight tapping, as described ${ }^{82}$. For tolerance, flies were put back onto regular food after a 30-min initial exposure, and were then re-exposed to the same vapor 4 hours later. Note that tolerance is not connected to initial sensitivity, and flies naively sensitive to ethanol-induced sedation can have no, or a reduced tolerance phenotype. Flies overexpressing hZip8 (and their sibling controls) were placed at $28^{\circ} \mathrm{C}$ for two days to increase the expression levels of the transgene, as we did not detect a phenotype when they were kept at $25^{\circ} \mathrm{C}$ (data not shown). Data from experimental and control flies were compared by Student's t-tests.

\section{Effects on other traits and diseases}

We queried SNPs against GWAS results included in PhenoScanner, to investigate cross-trait effects, extracting all association results with genome-wide significance at $P<5 \times 10^{-8}$ for all SNPs in high LD $\left(\mathrm{r}^{2} \geq 0.8\right)$ with the 46 sentinel novel SNPs, to highlight the loci with strongest evidence of association with other traits. At the gene level, overrepresentation enrichment analysis (ORA) with WebGestalt ${ }^{41}$ on the nearest genes to all alcohol intake loci was carried out.

The genetic correlations between alcohol consumption and 235 other traits and diseases were obtained in the online software LD Hub. LD hub is a centralized database of summary-level GWAS results and a web interface for LD score regression analysis

To estimate the potential causal effect of alcohol consumption-related variants on schizophrenia, we performed a Mendelian randomization analysis utilizing publicly available GWAS data on schizophrenia and the Mendelian randomization package in R. The effect was estimated using the inverse-variance weighted (IVM) method. Pleiotropy was tested by applying the MR-Egger regression method and heterogeneity statistics were obtained. In presence of heterogeneity the random effects inversevariance method was applied ${ }^{83}$.

\section{Genetic risk scores and percentage of variance explained}

We calculated an unbiased weighted genetic risk score in 14,004 unrelated participants in Airwave, an independent cohort with high quality HRC imputed genetic data ${ }^{33}$. We used as weights the beta coefficients of the meta-analysis. We assessed the association of the GRS with alcohol intake and calculated the alcohol consumption levels for individuals in the top vs the bottom quintiles of the 
875 distribution. To calculate the percent of variance of alcohol consumption explained by

876 genetic variants, we generated the residuals from a regression of alcohol consumption

877 in Airwave. We then fit a second linear model for the trait residuals with all novel and 878 known variants plus the top 10 principal components, and estimated the percentage

879 variance of the dependent variable explained by the variants.

880

881

882

883

884

885

886

887

888

889

890

891

892

893

894

895

896

897

898

899

900

901

902

903

904

905

906

907

908

909

910

911

912

913

914

915

916

917

\section{References}

64. Loh, P.R. et al. Efficient Bayesian mixed-model analysis increases association power in large cohorts. Nat Genet 47, 284-90 (2015).

65. Georgiopoulos, G. \& Evangelou, E. Power considerations for lambda inflation factor in meta-analyses of genome-wide association studies. Genet Res (Camb) 98, e9 (2016).

66. Winkler, T.W. et al. Quality control and conduct of genome-wide association meta-analyses. Nat Protoc 9, 1192-212 (2014).

67. Willer, C.J., Li, Y. \& Abecasis, G.R. METAL: fast and efficient meta-analysis of genomewide association scans. Bioinformatics 26, 2190-1 (2010).

68. Stacey, D. et al. RASGRF2 regulates alcohol-induced reinforcement by influencing mesolimbic dopamine neuron activity and dopamine release. Proc Natl Acad Sci U S A 109, 21128-33 (2012).

69. GTEx Consortium. The Genotype-Tissue Expression (GTEx) project. Nat Genet 45, 580-5 (2013).

70. Ramasamy, A. et al. Genetic variability in the regulation of gene expression in ten regions of the human brain. Nat Neurosci 17, 1418-1428 (2014).

71. Ongen, H., Buil, A., Brown, A.A., Dermitzakis, E.T. \& Delaneau, O. Fast and efficient QTL mapper for thousands of molecular phenotypes. Bioinformatics 32, 1479-85 (2016).

72. Storey, J.D. \& Tibshirani, R. Statistical significance for genomewide studies. Proc Natl Acad Sci U S A 100, 9440-5 (2003).

73. Shabalin, A.A. Matrix eQTL: ultra fast eQTL analysis via large matrix operations. Bioinformatics 28, 1353-8 (2012).

74. Brown, C.A. et al. Development, validation and application of a new fornix template for studies of aging and preclinical Alzheimer's disease. Neuroimage Clin 13, 106-115 (2017).

75. Diedrichsen, J. et al. Imaging the deep cerebellar nuclei: a probabilistic atlas and normalization procedure. Neuroimage 54, 1786-94 (2011).

76. Zhang, Y., Brady, M. \& Smith, S. Segmentation of brain MR images through a hidden Markov random field model and the expectation-maximization algorithm. IEEE Trans Med Imaging 20, 45-57 (2001).

77. Wager, T.D., Davidson, M.L., Hughes, B.L., Lindquist, M.A. \& Ochsner, K.N. Prefrontal-subcortical pathways mediating successful emotion regulation. Neuron 59, 1037-50 (2008).

78. Wager, T.D. et al. Brain mediators of cardiovascular responses to social threat: part I: Reciprocal dorsal and ventral sub-regions of the medial prefrontal cortex and heart-rate reactivity. Neuroimage 47, 821-35 (2009). 
919 79. Petersen, S.E. et al. UK Biobank's cardiovascular magnetic resonance protocol. J Cardiovasc Magn Reson 18, 8 (2016).

80. Bai, W. et al. Automated cardiovascular magnetic resonance image analysis with fully convolutional networks. J Cardiovasc Magn Reson 20, 65 (2018).

81. Linge, J. et al. Body Composition Profiling in the UK Biobank Imaging Study. Obesity (Silver Spring) (2018).

82. Peru, Y.C.d.P.R.L. et al. Adult neuronal Arf6 controls ethanol-induced behavior with Arfaptin downstream of Rac1 and RhoGAP18B. J Neurosci 32, 17706-13 (2012).

83. Dimou, N.L. \& Tsilidis, K.K. A Primer in Mendelian Randomization Methodology with a Focus on Utilizing Published Summary Association Data. Methods Mol Biol 1793, 211-230 (2018). 
bioRxiv preprint doi: https://doi.org/10.1101/453332; this version posted October 30, 2018. The copyright holder for this preprint (which was not certified by peer review) is the author/funder. All rights reserved. No reuse allowed without permission.

Table 1: Association results of 46 novel alcohol variants identified through the meta-analysis of UK Biobank and AlcGen and CHARGE+. Results are ordered by $P$-value of combined analysis.

\begin{tabular}{|c|c|c|c|c|c|c|c|c|c|c|c|c|c|c|}
\hline \multicolumn{6}{|c|}{ leadSNP } & \multicolumn{3}{|c|}{ Combined } & \multicolumn{3}{|c|}{ UKB } & \multicolumn{3}{|c|}{ AlcGen and CHARGE+ } \\
\hline Nearest_Gene & $\begin{array}{c}\text { Annotated } \\
\text { Gene }\end{array}$ & \begin{tabular}{|c|} 
rsID_LEAD_ \\
SNP
\end{tabular} & CP & EA & EAF & BETA & SE & $\mathbf{P}$ & BETA & SE & $\mathbf{P}$ & BETA & SE & $\mathbf{P}$ \\
\hline MAPT & STH & rs1991556 & $17: 44083402$ & A & 0.22 & -0.012 & 0.001 & $4.5 \mathrm{E}-23$ & -0.013 & 0.001 & $2.4 \mathrm{E}-21$ & -0.011 & 0.004 & $4.0 \mathrm{E}-03$ \\
\hline RP11-89K21.1 & SIX3 & rs1004787 & 2:45159091 & A & 0.54 & 0.009 & 0.001 & $6.7 \mathrm{E}-17$ & 0.009 & 0.001 & $1.1 \mathrm{E}-15$ & 0.007 & 0.003 & $1.4 \mathrm{E}-02$ \\
\hline SLC39A8 & SLC39A8 & rs13107325 & 4:103188709 & $\mathrm{T}$ & 0.07 & -0.016 & 0.002 & $1.3 \mathrm{E}-15$ & -0.017 & 0.002 & 4. $8 \mathrm{E}-16$ & -0.006 & 0.006 & $3.6 \mathrm{E}-01$ \\
\hline IZUMO1, RASIP1, FUT1 & IZUMO1 & rs838145 & 19:49248730 & A & 0.55 & -0.008 & 0.001 & $3.2 \mathrm{E}-15$ & -0.009 & 0.001 & $2.4 \mathrm{E}-15$ & -0.004 & 0.003 & $1.7 \mathrm{E}-01$ \\
\hline na & PSMD7 & rs1104608 & 16:73912588 & $c$ & 0.43 & -0.008 & 0.001 & $1.2 \mathrm{E}-14$ & -0.009 & 0.001 & 4.9E-15 & -0.003 & 0.003 & $2.5 \mathrm{E}-01$ \\
\hline MYBPC3 & MYBPC3 & rs2071305 & 11:47370957 & A & 0.69 & 0.009 & 0.001 & $4.5 \mathrm{E}-14$ & 0.009 & 0.001 & $3.9 \mathrm{E}-13$ & 0.007 & 0.003 & $3.1 \mathrm{E}-02$ \\
\hline na & DRD2 & rs7121986 & 11:113355444 & $\mathrm{T}$ & 0.37 & -0.008 & 0.001 & $6.2 \mathrm{E}-14$ & -0.008 & 0.001 & $1.3 \mathrm{E}-13$ & -0.005 & 0.003 & $1.1 \mathrm{E}-01$ \\
\hline na & DPP6 & rs6969458 & $7: 153489725$ & A & 0.47 & 0.008 & 0.001 & $6.4 \mathrm{E}-14$ & 0.008 & 0.001 & $1.3 \mathrm{E}-12$ & 0.007 & 0.003 & $1.5 \mathrm{E}-02$ \\
\hline RP11-308N 19.1 & ZNF462 & rs74424378 & 9:109331094 & $\mathrm{T}$ & 0.76 & 0.009 & 0.001 & $1.7 \mathrm{E}-13$ & 0.009 & 0.001 & 4.5E-13 & 0.006 & 0.003 & $8.4 \mathrm{E}-02$ \\
\hline ARHGAP15, AC096558.1, RP11-570L15.2 & ARHGAP15 & rs13024996 & $2: 144225215$ & A & 0.37 & -0.008 & 0.001 & $4.4 \mathrm{E}-13$ & -0.008 & 0.001 & $6.6 \mathrm{E}-13$ & -0.004 & 0.003 & $1.4 \mathrm{E}-01$ \\
\hline MLXIPL & MLXIPL & rs34060476 & 7:73037956 & $A$ & 0.87 & -0.011 & 0.002 & $5.0 \mathrm{E}-13$ & -0.012 & 0.002 & $1.4 \mathrm{E}-13$ & -0.004 & 0.004 & 4.1E-01 \\
\hline na & FAM178A & rs61873510 & $10: 102626510$ & $\mathrm{~T}$ & 0.33 & -0.008 & 0.001 & $5.1 \mathrm{E}-13$ & -0.008 & 0.001 & $9.8 \mathrm{E}-12$ & -0.008 & 0.003 & $1.7 \mathrm{E}-02$ \\
\hline FTO & FTO & rs1421085 & $16: 53800954$ & $\mathrm{~T}$ & 0.60 & 0.008 & 0.001 & $9.2 \mathrm{E}-13$ & 0.007 & 0.001 & $1.7 \mathrm{E}-10$ & 0.010 & 0.003 & $9.2 \mathrm{E}-04$ \\
\hline na & PMFBP1 & rs11648570 & $16: 72356964$ & $\mathrm{~T}$ & 0.89 & -0.012 & 0.002 & $2.1 \mathrm{E}-12$ & -0.011 & 0.002 & $1.5 \mathrm{E}-10$ & -0.013 & 0.005 & $3.4 \mathrm{E}-03$ \\
\hline ОТХ2, RP11-1085N6.6 & отХ2 & rs2277499 & 14:57271127 & $\mathrm{T}$ & 0.34 & -0.008 & 0.001 & $2.2 \mathrm{E}-12$ & -0.007 & 0.001 & $2.4 \mathrm{E}-09$ & -0.012 & 0.003 & $9.1 \mathrm{E}-05$ \\
\hline PDE4B & PDE4B & rs2310752 & 1:66392405 & A & 0.43 & -0.007 & 0.001 & $2.8 \mathrm{E}-12$ & -0.008 & 0.001 & $1.8 \mathrm{E}-11$ & -0.006 & 0.003 & $4.2 \mathrm{E}-02$ \\
\hline SERPINA1 & SERPINA1 & rs 112635299 & $14: 94838142$ & $\mathrm{~T}$ & 0.02 & -0.025 & 0.004 & $3.7 \mathrm{E}-12$ & -0.027 & 0.004 & $9.8 \mathrm{E}-12$ & -0.017 & 0.010 & $9.9 \mathrm{E}-02$ \\
\hline na & AJAP1 & rs780569 & 1:4569436 & A & 0.71 & -0.008 & 0.001 & $5.2 \mathrm{E}-12$ & -0.008 & 0.001 & $1.1 \mathrm{E}-11$ & -0.005 & 0.003 & $1.2 \mathrm{E}-01$ \\
\hline na & VRK2 & rs10496076 & 2:57942987 & $\mathrm{T}$ & 0.37 & -0.007 & 0.001 & $9.7 \mathrm{E}-12$ & -0.007 & 0.001 & $1.3 \mathrm{E}-09$ & -0.009 & 0.003 & $1.6 \mathrm{E}-03$ \\
\hline ACTR 10, C14orf37 & ACTR10 & rs71414193 & 14:58685301 & A & 0.19 & -0.009 & 0.001 & $1.8 \mathrm{E}-11$ & -0.008 & 0.001 & $5.8 \mathrm{E}-09$ & -0.013 & 0.004 & 4.5E-04 \\
\hline BEND4 & BEND4 & rs 16854020 & 4:42117559 & $A$ & 0.13 & 0.010 & 0.002 & $2.9 \mathrm{E}-11$ & 0.010 & 0.002 & $5.8 \mathrm{E}-09$ & 0.016 & 0.005 & $6.4 \mathrm{E}-04$ \\
\hline na & SORL1 & rs485425 & 11:121544984 & c & 0.45 & -0.007 & 0.001 & $6.1 \mathrm{E}-11$ & -0.007 & 0.001 & $7.3 \mathrm{E}-11$ & -0.004 & 0.003 & $1.9 \mathrm{E}-01$ \\
\hline SEZ6L2 & SEZ6L2 & rs 113443718 & $16: 29892184$ & A & 0.31 & -0.007 & 0.001 & $7.4 \mathrm{E}-11$ & -0.008 & 0.001 & 4.5E-11 & -0.003 & 0.003 & $2.9 \mathrm{E}-01$ \\
\hline CBX5, RP11-968A15.2 & CBX5 & rs57281063 & $12: 54660427$ & A & 0.41 & 0.007 & 0.001 & $7.9 \mathrm{E}-11$ & 0.007 & 0.001 & $1.8 \mathrm{E}-09$ & 0.007 & 0.003 & $1.2 \mathrm{E}-02$ \\
\hline na & TNRC6A & rs72768626 & $16: 24693048$ & $A$ & 0.94 & 0.014 & 0.002 & $9.7 \mathrm{E}-11$ & 0.015 & 0.002 & $1.7 \mathrm{E}-09$ & 0.014 & 0.006 & $1.8 \mathrm{E}-02$ \\
\hline SYT14 & SYT14 & rs 227179 & 1:210216731 & A & 0.59 & -0.007 & 0.001 & $1.1 \mathrm{E}-10$ & -0.007 & 0.001 & $1.4 \mathrm{E}-09$ & -0.006 & 0.003 & $2.8 \mathrm{E}-02$ \\
\hline TCF4 & TCF4 & rs9320010 & 18:53053897 & A & 0.60 & 0.007 & 0.001 & $1.1 \mathrm{E}-10$ & 0.007 & 0.001 & $1.6 \mathrm{E}-09$ & 0.007 & 0.003 & $2.2 \mathrm{E}-02$ \\
\hline SBK1 & NPIPB6 & rs2726034 & $16: 28336882$ & $\mathrm{~T}$ & 0.68 & 0.007 & 0.001 & $1.4 \mathrm{E}-10$ & 0.007 & 0.001 & $1.1 \mathrm{E}-09$ & 0.006 & 0.003 & 4.7E-02 \\
\hline ANKRD36 & ANKRD36 & rs13390019 & 2:97797680 & $\mathrm{T}$ & 0.87 & 0.010 & 0.002 & $1.6 \mathrm{E}-10$ & 0.011 & 0.002 & 7.0E-11 & 0.004 & 0.005 & 4.5E-01 \\
\hline na & ELAVL4 & rs7517344 & 1:50711961 & A & 0.17 & 0.009 & 0.001 & $1.9 \mathrm{E}-10$ & 0.008 & 0.001 & $2.5 \mathrm{E}-07$ & 0.016 & 0.004 & $2.1 \mathrm{E}-05$ \\
\hline LINC00461 & MEF2C & rs4916723 & 5:87854395 & A & 0.58 & 0.007 & 0.001 & $2.1 \mathrm{E}-10$ & 0.007 & 0.001 & $5.1 \mathrm{E}-10$ & 0.005 & 0.003 & $1.1 \mathrm{E}-01$ \\
\hline ARPC1B, ARPC1A & ARPC1B & rs10249167 & 7:98980879 & A & 0.87 & 0.010 & 0.002 & $2.9 \mathrm{E}-10$ & 0.009 & 0.002 & $8.1 \mathrm{E}-08$ & 0.015 & 0.004 & $3.8 \mathrm{E}-04$ \\
\hline EFNB3, WRAP53 & EFN B3 & rs7640 & $17: 7606722$ & C & 0.80 & 0.008 & 0.001 & $4.3 \mathrm{E}-10$ & 0.009 & 0.001 & $1.3 \mathrm{E}-09$ & 0.006 & 0.004 & $9.9 \mathrm{E}-02$ \\
\hline RP11-501C14.5 & IGF2BP1 & rs4794015 & $17: 47067826$ & A & 0.41 & 0.007 & 0.001 & $4.3 \mathrm{E}-10$ & 0.006 & 0.001 & $5.4 \mathrm{E}-08$ & 0.009 & 0.003 & $1.2 \mathrm{E}-03$ \\
\hline TCAP, PNMT, STARD3 & TCAP & rs1053651 & $17: 37822311$ & A & 0.27 & -0.007 & 0.001 & $1.1 \mathrm{E}-09$ & -0.008 & 0.001 & $8.4 \mathrm{E}-10$ & -0.003 & 0.003 & $2.8 \mathrm{E}-01$ \\
\hline na & AADAT & rs7698119 & 4:171070910 & A & 0.49 & -0.006 & 0.001 & $1.3 \mathrm{E}-09$ & -0.006 & 0.001 & $1.6 \mathrm{E}-07$ & -0.009 & 0.003 & $1.6 \mathrm{E}-03$ \\
\hline STAT6, AC023237.1 & STAT6 & rs12312693 & $12: 57511734$ & $\mathrm{~T}$ & 0.55 & -0.006 & 0.001 & $1.5 \mathrm{E}-09$ & -0.006 & 0.001 & 9.5E-09 & -0.005 & 0.003 & $5.6 \mathrm{E}-02$ \\
\hline SCN8A & SCN8A & rs7958704 & 12:51984349 & $\mathrm{T}$ & 0.41 & -0.006 & 0.001 & $1.6 \mathrm{E}-09$ & -0.006 & 0.001 & $1.7 \mathrm{E}-08$ & -0.006 & 0.003 & $3.5 \mathrm{E}-02$ \\
\hline ACSS3 & ACSS3 & rs11114787 & 12:81595700 & $\mathrm{T}$ & 0.27 & 0.007 & 0.001 & $2.0 \mathrm{E}-09$ & 0.007 & 0.001 & 2.7E-08 & 0.007 & 0.003 & $2.4 \mathrm{E}-02$ \\
\hline RP11-32K4.1 & BHLHE22 & rs2356369 & 8:64956882 & $\mathrm{T}$ & 0.52 & -0.006 & 0.001 & $2.0 \mathrm{E}-09$ & -0.006 & 0.001 & 4.1E-08 & -0.007 & 0.003 & $1.6 \mathrm{E}-02$ \\
\hline ZRANB2-AS2 & ZRANB2 & rs12031875 & 1:71585097 & A & 0.82 & -0.008 & 0.001 & $2.2 \mathrm{E}-09$ & -0.008 & 0.001 & $7.6 \mathrm{E}-08$ & -0.010 & 0.004 & $8.7 \mathrm{E}-03$ \\
\hline MSANTD 1, HTT & MSANTD1 & rs 12646808 & 4:3249828 & $\mathrm{T}$ & 0.66 & 0.007 & 0.001 & $2.4 \mathrm{E}-09$ & 0.007 & 0.001 & $1.1 \mathrm{E}-09$ & 0.002 & 0.003 & 4.7E-01 \\
\hline TENM2 & TENM2 & rs10078588 & $5: 166816176$ & A & 0.52 & 0.006 & 0.001 & $2.5 \mathrm{E}-09$ & 0.006 & 0.001 & 4.3E-08 & 0.007 & 0.003 & $1.9 \mathrm{E}-02$ \\
\hline IGSF9B & IGSF9B & rs748919 & $11: 133783232$ & $\mathrm{~T}$ & 0.79 & 0.008 & 0.001 & $3.3 \mathrm{E}-09$ & 0.008 & 0.001 & $1.0 \mathrm{E}-08$ & 0.005 & 0.003 & $1.1 \mathrm{E}-01$ \\
\hline AC010967.2 & GPR75-ASB3 & rs785293 & 2:53023304 & $A$ & 0.57 & -0.006 & 0.001 & $3.3 \mathrm{E}-09$ & -0.006 & 0.001 & $3.2 \mathrm{E}-08$ & -0.006 & 0.003 & $3.8 \mathrm{E}-02$ \\
\hline BDNF, RP11-587D21.4 & BDNF & rs988748 & $11: 27724745$ & C & 0.21 & -0.008 & 0.001 & $4.4 \mathrm{E}-09$ & -0.007 & 0.001 & $1.2 \mathrm{E}-07$ & -0.010 & 0.004 & $8.3 \mathrm{E}-03$ \\
\hline
\end{tabular}

SNP: Single Nucleotide polymorphism; LocusName: Nearest Gene; rsID_LEAD_SNP: Rs ID number of the lead SNP; CP: Chromosome/Position (build hg19/37); EA: Effect allele of the discovered SNP; EAF: Frequency of the effect allele; BETA_comb: Effect size in meta-analysis; SE_comb; Standard Error of the effect in meta-analysis; P_comb: Meta-analysis P-value; BETA_UKB: Effect size in UK Biobank analysis; SE_UKB: Standard Error of the effect in the UK Biobank analysis; P_UKB: UK Biobank analysis P-value;BETA_AlcGenCHARGE+: Effect size in the AlcGen meta-analysis; SE_AlcGenCHARGE+: Standard Error of the effect in the AlcGen meta-analysis; P_AlcGenCHARGE+: AlcGen meta-analysis P-value 